\title{
A Study of Dark Matter with Spiral Galaxy Rotation Curves
}

\author{
Bruce Hoeneisen \\ Universidad San Francisco de Quito, Quito, Ecuador \\ Email: bruce1@fnal.gov
}

How to cite this paper: Hoeneisen, B. (2019) A Study of Dark Matter with Spiral Galaxy Rotation Curves. International Journal of Astronomy and Astrophysics, 9, 71-96.

https://doi.org/10.4236/ijaa.2019.92007

Received: April 2, 2019

Accepted: May 2, 2019

Published: May 5, 2019

Copyright (c) 2019 by author(s) and Scientific Research Publishing Inc. This work is licensed under the Creative Commons Attribution International License (CC BY 4.0).

http://creativecommons.org/licenses/by/4.0/ (c) (i) Open Access

\section{Abstract}

To constrain the properties of dark matter, we study spiral galaxy rotation curves measured by the THINGS collaboration. A model that describes a mixture of two self-gravitating non-relativistic ideal gases, "baryons" and "dark matter", reproduces the measured rotation curves within observational uncertainties. The model has four parameters that are obtained by minimizing a $\chi^{2}$ between the measured and calculated rotation curves. From these four parameters, we calculate derived galaxy parameters. We find that dark matter satisfies the Boltzmann distribution. The onset of Fermi-Dirac or Bose-Einstein degeneracy obtains disagreement with observations and we determine, with $99 \%$ confidence, that the mass of dark matter particles is $m_{h}>16 \mathrm{eV}$ if fermions, or $m_{h}>45 \mathrm{eV}$ if bosons. We measure the root-mean-square velocity of dark matter particles in the spiral galaxies. This observable is of cosmological origin and allows us to obtain the root-mean-square velocity of dark matter particles in the early universe when perturbations were still linear. Extrapolating to the past we obtain the expansion parameter at which dark matter particles become non-relativistic: $a_{h \mathrm{NR}}=[4.17 \pm 0.34$ (stat) \pm 2.50 (syst) $] \times 10^{-6}$. Knowing $\Omega_{c} \rho_{\text {crit }}$ we then obtain the dark matter particle mass $m_{h}=69.0 \pm 4.2$ (stat) \pm 31.0 (syst) $\mathrm{eV}$, and the ratio of dark matter-to-photon temperature $T_{h} / T=0.389 \pm 0.008$ (stat) \pm 0.058 (syst) after $e^{+} e^{-}$annihilation while dark matter remains ultra-relativistic. We repeat these measurements with ten galaxies with masses that span three orders of magnitude, and angular momenta that span five orders of magnitude, and obtain fairly consistent results. We conclude that dark matter was once in thermal equilibrium with the (pre?) Standard Model particles (hence the observed Boltzmann distribution) and then decoupled from the Standard Model and from self-annihilation at temperatures above $m_{\mu}$. These results disfavor models with freeze-out or freeze-in. We also measure 
the primordial amplitude of vector modes, and constrain the baryon-dark matter cross-section: $\sigma_{h b} \lesssim 2 \times 10^{-26} \mathrm{~cm}^{2}$. Finally, we consider sterile Majorana neutrinos as a dark matter candidate.

\section{Keywords}

Dark Matter, Spiral Galaxies, Majorana Neutrinos

\section{Introduction}

The dark matter density in the core of spiral galaxies can exceed $10^{7}$ times the mean dark matter density of the Universe. To learn about the properties of dark matter, we study the rotation curves of spiral galaxies measured by the THINGS collaboration [1]. The galaxies studied are in a stationary state, i.e. they have pulled away from the expansion of the Universe.

We use the standard notation for cosmology as defined in [2], and the values of the cosmological parameters presented therein. Sometimes we use units with $\hbar=1$ and $c=1$ as is customary.

\section{A Model of Spiral Galaxy Rotation Curves}

Figure 1 presents rotation curves of the spiral galaxy NGC 3198 measured by the THINGS collaboration [1]. The observed rotation curve $v_{\text {obs }}(r)$ has contributions from baryons $v_{b}(r)$ (stars in the disk and bulge, and gas), and from the halo of dark matter $v_{h}(r)$ :

$$
v_{\text {obs }}^{2} \equiv v^{2}=v_{b}^{2}+v_{h}^{2}, \quad v_{b}^{2}=v_{\text {disk }}^{2}+v_{\text {bulge }}^{2}+v_{\text {gas }}^{2} .
$$

These rotation velocities correspond, by definition, to test particles in circular orbits of radius $r$ in the plane of the galaxy. There is good agreement between the THINGS analysis and previous measurements by K. Begeman [3]. The mass distribution of stars is obtained from measurements of surface brightness in the $3.6 \mu \mathrm{m}$ band and models of the mass-to-light ratio $\Upsilon_{*}^{3.6}$. The mass distribution of gas is obtained from $H_{I}$ maps. The uncertainties of $v(r)$ are from the THINGS observations and analysis [1]. We estimate the uncertainties of $v_{b}(r)$ from the scatter between 4 fits presented by the THINGS collaboration: "ISO, free", "ISO, fixed", "NFK, free", and "NFK, fixed" [1]. These fits assume either the Navarro, Frenk and White (NFK) halo model [4], or the pseudo-isothermal-gas (ISO) model.

From the measured rotation curves $v(r)$ and $v_{b}(r)$ we can obtain directly $g_{b}=-v_{b}^{2} / r$ and $g_{h}=-\left(v^{2}-v_{b}^{2}\right) / r$. From $g_{h}=-M_{h}(r) G / r^{2} \quad$ (assuming spherical symmetry) we can obtain

$$
\rho_{h}=\frac{1}{4 \pi G r^{2}} \frac{\mathrm{d}\left(r v_{h}^{2}\right)}{\mathrm{d} r},
$$

and similarly for $\rho_{b}$. We use these equations as cross-checks. Note that $\rho_{h}(r)$ 

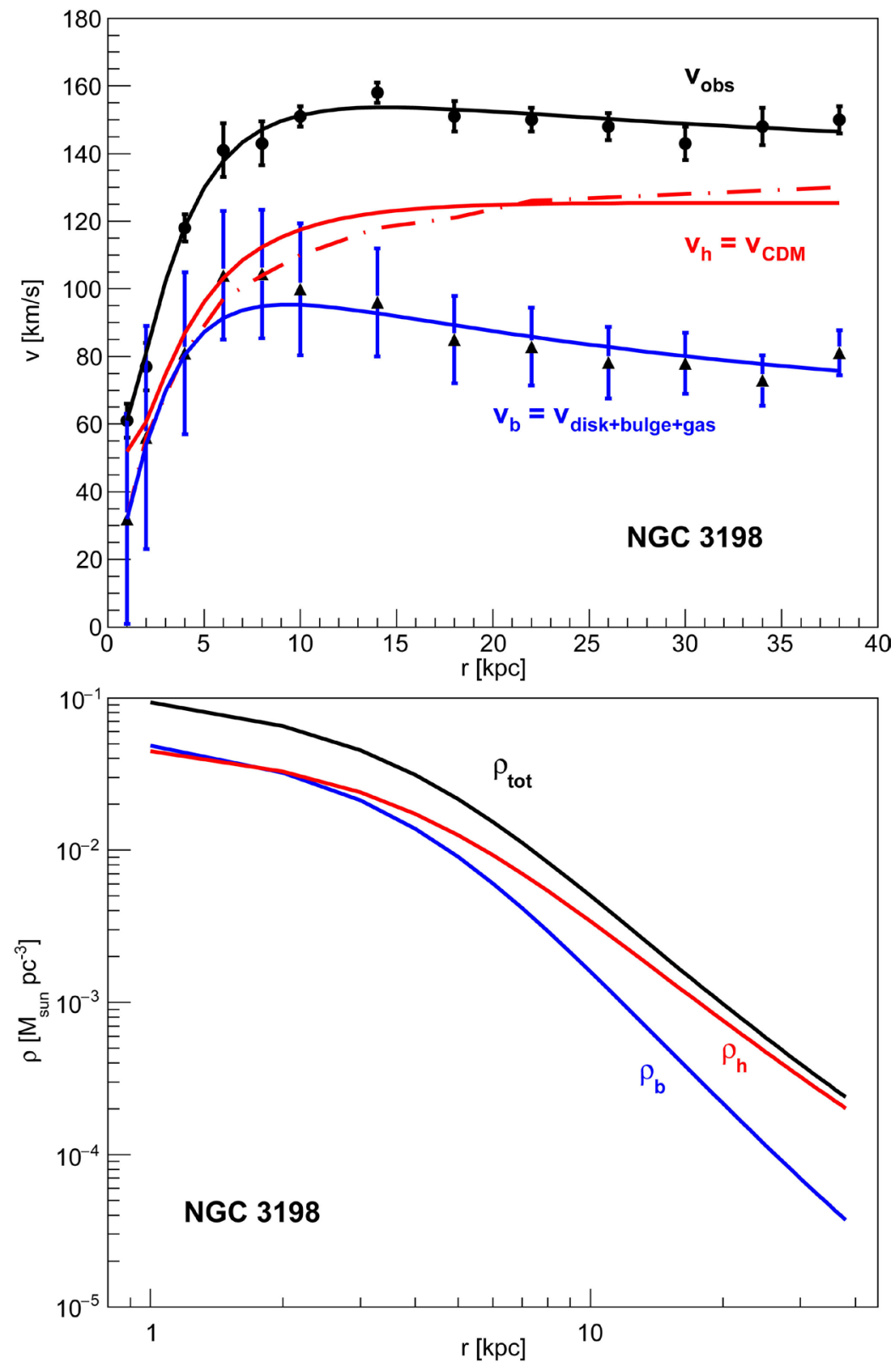

Figure 1. Top figure: Observed rotation curve $v_{\text {obs }}(r)$ (dots) and the baryon contribution $v_{b}(r)$ (triangles) of galaxy NGC 3198 obtained by the THINGS collaboration [1]. The "ISO, free" fit obtains $v_{h}(r)$ (dash-dotted line) [1]. The solid lines are obtained by numerical integration as explained in the text. The fitted parameters are given in Table 1. Bottom figure: Mass densities of baryons and of dark matter of galaxy NGC 3198 obtained by numerical integration as explained in the text.

and $\rho_{b}(r)$ are insensitive to whether or not the dark matter is rotating.

In the present analysis we would like to go further, i.e. constrain the equation of state of dark matter. To this end we compare the measured rotation curves with the following simplified model of a mixture of two self-gravitating non-relativistic ideal gases: "baryons" and "dark matter". We assume that the interactions between baryons and dark matter can be neglected. 
Table 1. Fitted parameters with $\kappa_{b}=0$, and $\kappa_{h}=0$. The last column shows the $\chi^{2}$ of the fit and the number of degrees of freedom. The fits are presented in Figures 1-10.

\begin{tabular}{cccccc}
\hline Galaxy & $\left\langle v_{r h}^{2}\right\rangle^{1 / 2}$ & $\left\langle v_{r b}^{2}\right\rangle^{1 / 2}$ & $\rho_{h}\left(r_{\min }\right)$ & $\rho_{b}\left(r_{\min }\right)$ & \\
& {$[\mathrm{km} / \mathrm{s}]$} & {$[\mathrm{km} / \mathrm{s}]$} & {$\left[10^{-2} M_{\odot} \cdot \mathrm{pc}^{-3}\right]$} & {$\left[10^{-2} M_{\odot} \cdot \mathrm{pc}^{-3}\right]$} & $\chi^{2} /$ d.f. \\
\hline NGC 2403 & $101 \pm 3$ & $63.4 \pm 1.8$ & $7.5 \pm 1.4$ & $30.4 \pm 6.2$ & $7.2 / 20$ \\
NGC 2841 & $220 \pm 3$ & $168 \pm 3$ & $9.3 \pm 0.7$ & $9.7 \pm 1.2$ & $22.1 / 22$ \\
NGC 2903 & $142 \pm 3$ & $117 \pm 3$ & $14.6 \pm 2.1$ & $25.2 \pm 3.1$ & $29.0 / 24$ \\
NGC 2976 & $129 \pm 177$ & $49 \pm 6$ & $4.0 \pm 2.7$ & $17.0 \pm 3.7$ & $9.8 / 16$ \\
NGC 3198 & $104 \pm 3$ & $90 \pm 3$ & $4.5 \pm 0.8$ & $4.9 \pm 1.2$ & $8.3 / 22$ \\
NGC 3521 & $153 \pm 10$ & $136 \pm 5$ & $22.9 \pm 8.6$ & $43.4 \pm 11.8$ & $6.8 / 20$ \\
NGC 3621 & $126 \pm 5$ & $78.8 \pm 1.0$ & $2.6 \pm 0.5$ & $26.6 \pm 1.5$ & $13.2 / 22$ \\
DDO 154 & $36.5 \pm 3.7$ & $22.3 \pm 1.7$ & $1.3 \pm 0.3$ & $1.9 \pm 0.8$ & $7.5 / 18$ \\
NGC 5055 & $144 \pm 4$ & $130 \pm 3$ & $28.2 \pm 6.8$ & $41.4 \pm 10.1$ & $19.2 / 24$ \\
NGC 7793 & $85.5 \pm 5.0$ & $52.2 \pm 1.5$ & $8.0 \pm 1.6$ & $29.7 \pm 4.4$ & $36.3 / 16$ \\
\hline
\end{tabular}

$$
\begin{gathered}
\nabla \cdot \boldsymbol{g}_{b}=-4 \pi G \rho_{b}, \quad \nabla \cdot \boldsymbol{g}_{h}=-4 \pi G \rho_{h}, \\
\boldsymbol{g}=\boldsymbol{g}_{b}+\boldsymbol{g}_{h}, \quad g_{b} \equiv-\frac{v_{b}^{2}}{r}, \quad g_{h} \equiv-\frac{v_{h}^{2}}{r}, \quad v^{2} \equiv v_{b}^{2}+v_{h}^{2}, \\
\nabla P_{b}=\rho_{b}\left(\boldsymbol{g}+\kappa_{b} \frac{v^{2}}{r} \hat{\boldsymbol{e}}_{r}\right), \quad \nabla P_{h}=\rho_{h}\left(\boldsymbol{g}+\kappa_{h} \frac{v^{2}}{r} \hat{\boldsymbol{e}}_{r}\right), \\
P_{b}=\left\langle v_{r b}^{2}\right\rangle \rho_{b} \text { and } P_{h}=\left\langle v_{r h}^{2}\right\rangle \rho_{h}\left(1 \pm \rho_{h} \frac{\pi^{3 / 2} \hbar^{3}}{2\left\langle v_{r h}^{2}\right\rangle^{3 / 2} N_{f, b} m_{h}^{4}}+\cdots\right) .
\end{gathered}
$$

$\left\langle v_{r b}^{2}\right\rangle$ and $\left\langle v_{r h}^{2}\right\rangle$ are the mean-square of the velocity components in the radial direction $r$ of baryons and dark matter respectively. Newton's Equation (3) determine the gravitational field $\boldsymbol{g}(r)=\boldsymbol{g}_{b}(r)+\boldsymbol{g}_{h}(r)$ due to the densities $\rho_{b}(r)$ of baryons and $\rho_{h}(r)$ of dark matter. Equations (5) express momentum conservation and are valid even for collisionless gases. The centrifugal acceleration terms proportional to $\kappa_{b}$ and $\kappa_{h}$ are inserted to study the rotation of baryons and dark matter.

We consider dark matter to be a mixture of interacting or non-interacting particles of masses $m_{h i}$. Let $n_{h i}\left(r, v_{r}\right) \mathrm{d} v_{r}$ be the number density of dark matter particles of mass $m_{h i}$ with radial component of velocity between $v_{r}$ and $v_{r}+\mathrm{d} v_{r}$. Then

$$
\rho_{h}=\sum_{i} m_{h i} \int_{0}^{\infty} n_{h i}\left(r, v_{r}\right) \mathrm{d} v_{r}, \quad P_{h} \equiv\left\langle v_{r h}^{2}\right\rangle \rho_{h},
$$

where

$$
\left\langle v_{r h}^{2}\right\rangle \equiv \frac{\sum_{i} m_{h i} \int_{0}^{\infty} v_{r}^{2} \cdot n_{h i}\left(r, v_{r}\right) \mathrm{d} v_{r}}{\sum_{i} m_{h i} \int_{0}^{\infty} n_{h i}\left(r, v_{r}\right) \mathrm{d} v_{r}}
$$


and similarly for baryons. The pressure $P_{h}(r)$ is the momentum component in the radial direction in the galactic plane per unit time traversing unit area at $r$ with $v_{r}>0$.

Equations (6) are the equations of state of the gasses. For the dark matter halo we have included a term due to the onset of degeneracy of fermions (upper signs) or bosons (lower signs). $N_{f}\left(N_{b}\right)$ is the number of fermion (boson) degrees of freedom. We take $N_{f}=2$ for one flavor of spin-up and spin-down sterile Majorana neutrinos. Results can be amended for other cases. For bosons we take $N_{b}=1$. Fermi-Dirac and Bose-Einstein degeneracy will be considered in Section 7. For now we set $m_{h}$ to some large value, e.g. $m_{h}=500 \mathrm{eV}$, so the last term in Equation (6) is negligible.

We integrate numerically Equations (3) to (6) from $r_{\min }$ to $r_{\max }$ along a radial direction in the galactic plane. Hence $\nabla P_{h}=\hat{\boldsymbol{e}}_{r} \mathrm{~d} P_{h} / \mathrm{d} r$ and $\nabla \cdot \boldsymbol{g}_{h}=\left(1 / r^{2}\right) \mathrm{d}\left(r^{2} g_{h}\right) / \mathrm{d} r$, and similarly for baryons.

Variables $\kappa_{b}$ and $\left\langle v_{r b}^{2}\right\rangle$, and also $\kappa_{h}$ and $\left\langle v_{r h}^{2}\right\rangle$, occur in the combinations

$$
\left\langle v_{r b}^{2}\right\rangle^{\prime} \equiv \frac{\left\langle v_{r b}^{2}\right\rangle}{1-\kappa_{b}},\left\langle v_{r h}^{2}\right\rangle^{\prime} \equiv \frac{\left\langle v_{r h}^{2}\right\rangle}{1-\kappa_{h}},
$$

if the last term in (6) is negligible. To lift this degeneracy and obtain these variables separately, we need information in addition to the galaxy rotation curves.

We fit four parameters to minimize the $\chi^{2}$ between the measured and calculated rotation velocities $v(r)$ and $v_{b}(r)$ : the starting densities $\rho_{h}\left(r_{\min }\right)$ and $\rho_{b}\left(r_{\min }\right)$, and the "reduced" root-mean-square radial velocities $\left\langle v_{r h}^{2}\right\rangle^{\prime}$, and $\left\langle v_{r b}^{2}\right\rangle^{\prime}$. These parameters are boundary conditions at $r \rightarrow 0$ and $r \rightarrow \infty$. Assuming $\left\langle v_{r h}^{2}\right\rangle^{\prime}$ and $\left\langle v_{r b}^{2}\right\rangle^{\prime}$ are independent of $r$ we obtain fits to the galaxy rotation curves that are in agreement with observations, within the experimental uncertainties, as shown in Figures 1-10, and in Table 1. The parameter correlation coefficients for galaxy NGC 3198 are shown in Table 2.

\section{Derived Galaxy Parameters}

We define $M_{b}(r)$ as the baryon mass contained within $r$, and similarly for $M_{h}(r)$. Equations (3) to (6) allow the definition of several galaxy parameters: the "equal density radius" $r_{\text {ed }}$ with $\rho_{b}\left(r_{\text {ed }}\right)=\rho_{h}\left(r_{\text {ed }}\right)$, the "galaxy proper radius"

Table 2. Correlation coefficients of the four fitted parameters in Table 1 for galaxy NGC 3198.

\begin{tabular}{ccccc}
\hline & $\left\langle v_{r h}^{2}\right\rangle^{1 / 2}$ & $\left\langle v_{r b}^{2}\right\rangle^{1 / 2}$ & $\rho_{h}\left(r_{\min }\right)$ & $\rho_{b}\left(r_{\min }\right)$ \\
\hline$\left\langle v_{r h}^{2}\right\rangle^{1 / 2}$ & 1.000 & -0.833 & -0.802 & 0.860 \\
$\left\langle v_{r b}^{2}\right\rangle^{1 / 2}$ & -0.833 & 1.000 & 0.775 & -0.831 \\
$\rho_{h}\left(r_{\min }\right)$ & -0.802 & 0.775 & 1.000 & -0.639 \\
$\rho_{b}\left(r_{\min }\right)$ & 0.860 & -0.831 & -0.639 & 1.000 \\
\hline
\end{tabular}


$r_{g}$ at which $M_{b}\left(r_{g}\right) / M_{h}\left(r_{g}\right)=\Omega_{b} / \Omega_{c}$, and the corresponding "primordial comoving radius" $r_{c}$ defined by $M_{b} \equiv \frac{4}{3} \pi r_{c}^{3} \Omega_{b} \rho_{\text {crit }}$. The "baryon mass" $M_{b}$ converges, so we define $M_{b} \equiv M_{b}\left(r_{g}\right)$. In the early Universe, when the perturbation that formed the galaxy was still linear, the matter of the galaxy was contained in the expanding sphere of proper radius $r_{g}$, and comoving radius $r_{c}$ at
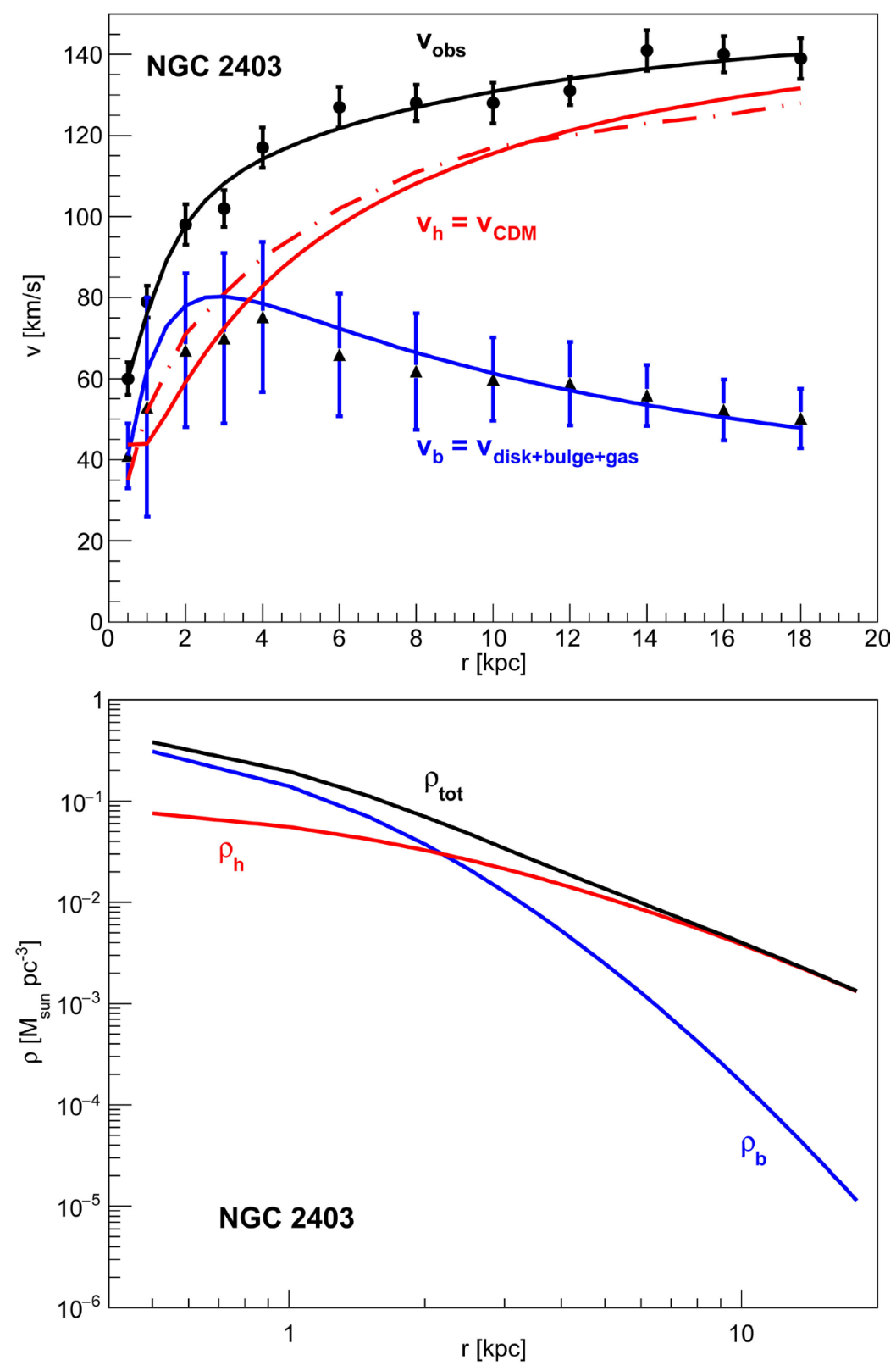

Figure 2. Top figure: Observed rotation curve $v_{\text {obs }}(r)$ (dots) and the baryon contribution $v_{b}(r)$ (triangles) of galaxy NGC 2403 obtained by the THINGS collaboration [1]. The "NFW, free" fit obtains $v_{h}(r)$ (dash-dotted line) [1]. The solid lines are obtained by numerical integration for non-degenerate dark matter as explained in the text. The fitted parameters are given in Table 1. Bottom figure: Mass densities of baryons and of dark matter of galaxy NGC 2403 obtained by numerical integration as explained in the text. 

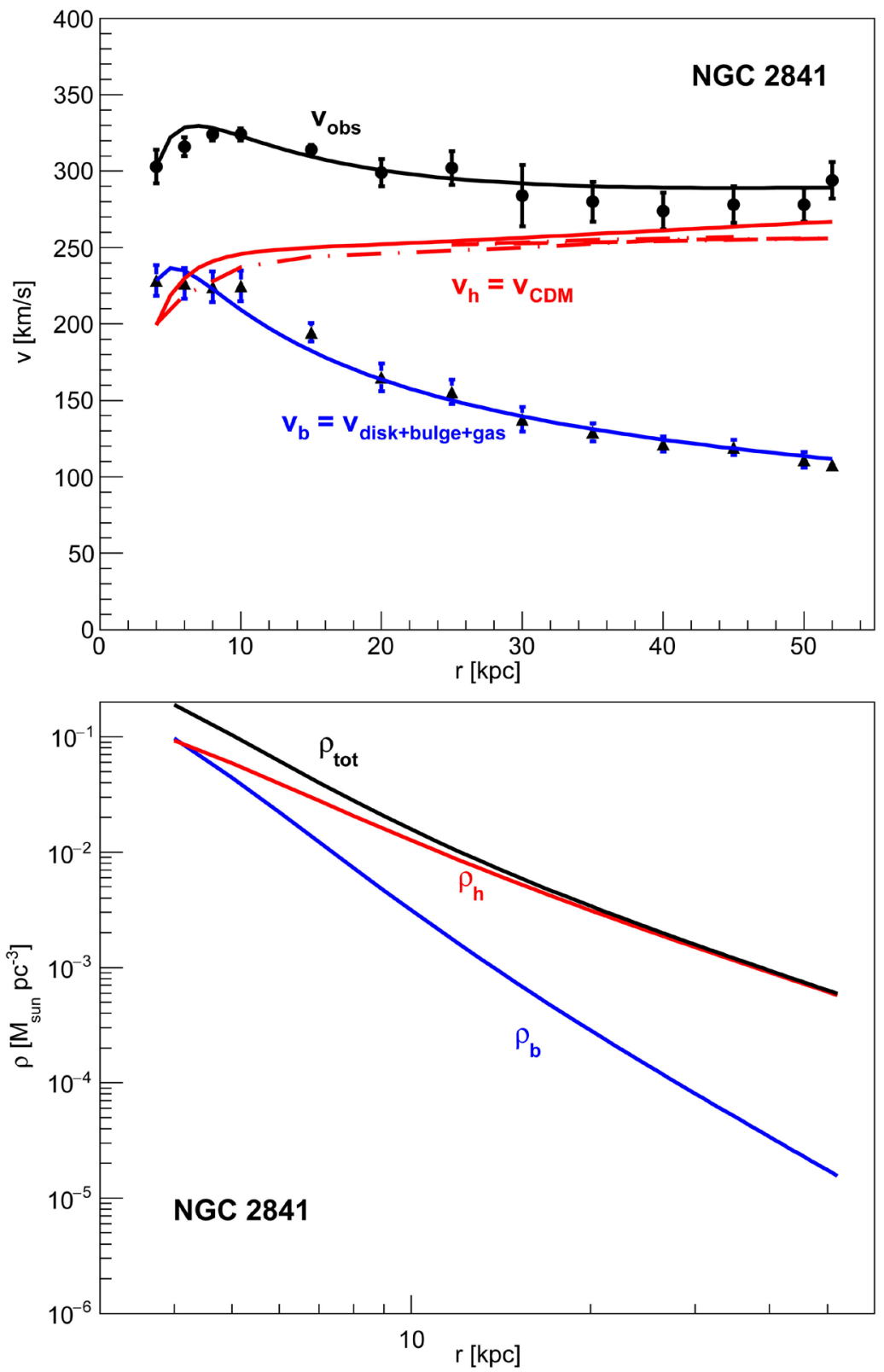

Figure 3. Top figure: Observed rotation curve $v_{\mathrm{obs}}(r)$ (dots) and the baryon contribution $v_{b}(r)$ (triangles) of galaxy NGC 2841 obtained by the THINGS collaboration [1]. The "ISO, free" fit obtains $v_{h}(r)$ (dash-dotted line) [1]. The solid lines are obtained by numerical integration as explained in the text. The fitted parameters are given in Table 1. Bottom figure: Mass densities of baryons and of dark matter of galaxy NGC $2841 \mathrm{ob}-$ tained by numerical integration as explained in the text.

time $t_{g}$. At time $t_{g}$ the expansion parameter is $r_{g} / r_{c}$. The results, obtained with the four fitted parameters listed in Table 1, are presented in Table 3. The angular momenta of baryons $L_{b}$ inside $r_{g}$ are given in Table 4.

\section{Estimate of $\kappa_{b}$}

As an example, let us consider the spiral galaxy NGC 2403. A distribution of ve- 
locities along a particular line of sight is presented in figure 3 of [1]. The central value is $\approx 186 \mathrm{~km} / \mathrm{s}$, and the standard deviation is $\approx 11 \mathrm{~km} / \mathrm{s}$. So we estimate $\sqrt{\kappa_{b}} v /\left\langle v_{r b}^{2}\right\rangle^{1 / 2} \approx 186 / 11$. From Table $1,\left\langle v_{r b}^{2}\right\rangle^{1 / 2} \equiv\left\langle v_{r b}^{2}\right\rangle^{1 / 2} /\left(1-\kappa_{b}\right)^{1 / 2}=63.4 \pm 1.8$ $\mathrm{km} / \mathrm{s}$, and from Figure $2, v \approx 140 \mathrm{~km} / \mathrm{s}$. From these relations we obtain $\kappa_{b} \approx 0.98$, so the trajectories of stars and gas are approximately circular.

\section{Estimate of $\kappa_{h}$}

Consider an expanding sphere of proper radius $r_{g}$ and comoving radius $r_{c}$ at time $t_{g}$ containing the matter that will become spiral galaxy NGC 2403. We assume adiabatic primordial perturbations, so the velocity fields of baryons and

Table 3. Galaxy parameters obtained by numerical integration with the fitted parameters in Table 1. See Section 3 for definitions.

\begin{tabular}{cccccc}
\hline Galaxy & $M_{b}\left[10^{10} M_{\odot}\right]$ & $r_{\text {ed }}[\mathrm{kpc}]$ & $r_{g}[\mathrm{kpc}]$ & $r_{c}[\mathrm{Mpc}]$ & $t_{g}[\mathrm{Gyr}]$ \\
\hline NGC 2403 & $0.9 \times e^{ \pm 0.22}$ & $2.2 \times e^{ \pm 0.28}$ & $13.6 \times e^{ \pm 0.24}$ & $0.7 \times e^{ \pm 0.07}$ & $0.047 \times e^{ \pm 0.25}$ \\
NGC 2841 & $15 \times e^{ \pm 0.13}$ & $4.1 \times e^{ \pm 0.12}$ & $48.5 \times e^{ \pm 0.18}$ & $1.8 \times e^{ \pm 0.04}$ & $0.077 \times e^{ \pm 0.20}$ \\
NGC 2903 & $10 \times e^{ \pm 0.46}$ & $3.5 \times e^{ \pm 0.20}$ & $74 \times e^{ \pm 0.47}$ & $1.6 \times e^{ \pm 0.15}$ & $0.18 \times e^{ \pm 0.48}$ \\
NGC 2976 & $0.4 \times e^{ \pm 0.58}$ & $1.8 \times e^{ \pm 0.41}$ & $6.3 \times e^{ \pm 0.47}$ & $0.5 \times e^{ \pm 0.19}$ & $0.022 \times e^{ \pm 0.45}$ \\
NGC 3198 & $9 \times e^{ \pm 1.0}$ & $1.9 \times e^{ \pm 1.0}$ & $128 \times e^{ \pm 1.0}$ & $1.5 \times e^{ \pm 0.34}$ & $0.42 \times e^{ \pm 1.0}$ \\
NGC 3521 & $69 \times e^{ \pm 1.8}$ & $5.0 \times e^{ \pm 0.87}$ & $403 \times e^{ \pm 2.0}$ & $3.0 \times e^{ \pm 0.60}$ & $0.86 \times e^{ \pm 1.5}$ \\
NGC 3621 & $3.9 \times e^{ \pm 0.18}$ & $5.8 \times e^{ \pm 0.15}$ & $38 \times e^{ \pm 0.20}$ & $1.1 \times e^{ \pm 0.06}$ & $0.11 \times e^{ \pm 0.22}$ \\
DDO 154 & $0.07 \times e^{ \pm 0.48}$ & $1.1 \times e^{ \pm 0.65}$ & $7.0 \times e^{ \pm 0.46}$ & $0.3 \times e^{ \pm 0.16}$ & $0.062 \times e^{ \pm 0.46}$ \\
NGC 5055 & $62 \times e^{ \pm 1.3}$ & $2.3 \times e^{ \pm 0.77}$ & $416 \times e^{ \pm 1.5}$ & $2.9 \times e^{ \pm 0.42}$ & $0.95 \times e^{ \pm 1.5}$ \\
NGC 7793 & $0.5 \times e^{ \pm 0.23}$ & $1.6 \times e^{ \pm 0.19}$ & $10 \times e^{ \pm 0.27}$ & $0.6 \times e^{ \pm 0.08}$ & $0.04 \times e^{ \pm 0.30}$ \\
\hline
\end{tabular}

Table 4. Baryon angular momentum $L_{b}$ within radius $r_{g}$ obtained by numerical integration (assuming $\kappa_{b}=0.98$ ), and limits on $m_{h}$ at $99 \%$ confidence. $r_{g}$ is defined in Section 3 .

\begin{tabular}{cccc}
\hline Galaxy & $\log _{10}\left\{L_{b} /\left[M_{\odot} \mathrm{km} / \mathrm{s} \cdot \mathrm{pc}\right]\right\}$ & Fermion $m_{h}>$ & Boson $m_{h}>$ \\
\hline NGC 2403 & $12.63 \pm 0.17$ & $18 \mathrm{eV}$ & no limit \\
NGC 2841 & $14.65 \pm 0.15$ & $12 \mathrm{eV}$ & $35 \mathrm{eV}$ \\
NGC 2903 & $14.61 \pm 0.44$ & $18 \mathrm{eV}$ & $40 \mathrm{eV}$ \\
NGC 2976 & $11.93 \pm 0.43$ & no limit & no limit \\
NGC 3198 & $14.76 \pm 0.94$ & $16 \mathrm{eV}$ & $45 \mathrm{eV}$ \\
NGC 3521 & $16.37 \pm 1.58$ & $10 \mathrm{eV}$ & no limit \\
NGC 3621 & $13.73 \pm 0.15$ & no limit & no limit \\
DDO 154 & $10.86 \pm 0.36$ & $30 \mathrm{eV}$ & no limit \\
NGC 5055 & $16.32 \pm 1.07$ & no limit & no limit \\
NGC 7793 & $12.13 \pm 0.19$ & $25 \mathrm{eV}$ & no limit \\
\hline
\end{tabular}


of dark matter are the same. Then the angular momenta of baryons and dark matter in the sphere of proper radius $r_{g}$ are in the ratio $L_{b} / L_{h}=M_{b} / M_{h}\left(r_{g}\right)=\Omega_{b} / \Omega_{h}$. According to the model of hierarchical formation of galaxies [5] [6], the sphere of radius $r_{g}$ expands, reaches maximum expansion, and then collapses to form a galaxy, see figure 5 of Reference [6]. The angular momenta $L_{b}$ and $L_{h}$ are conserved if we neglect mixing. The density becomes approximately proportional
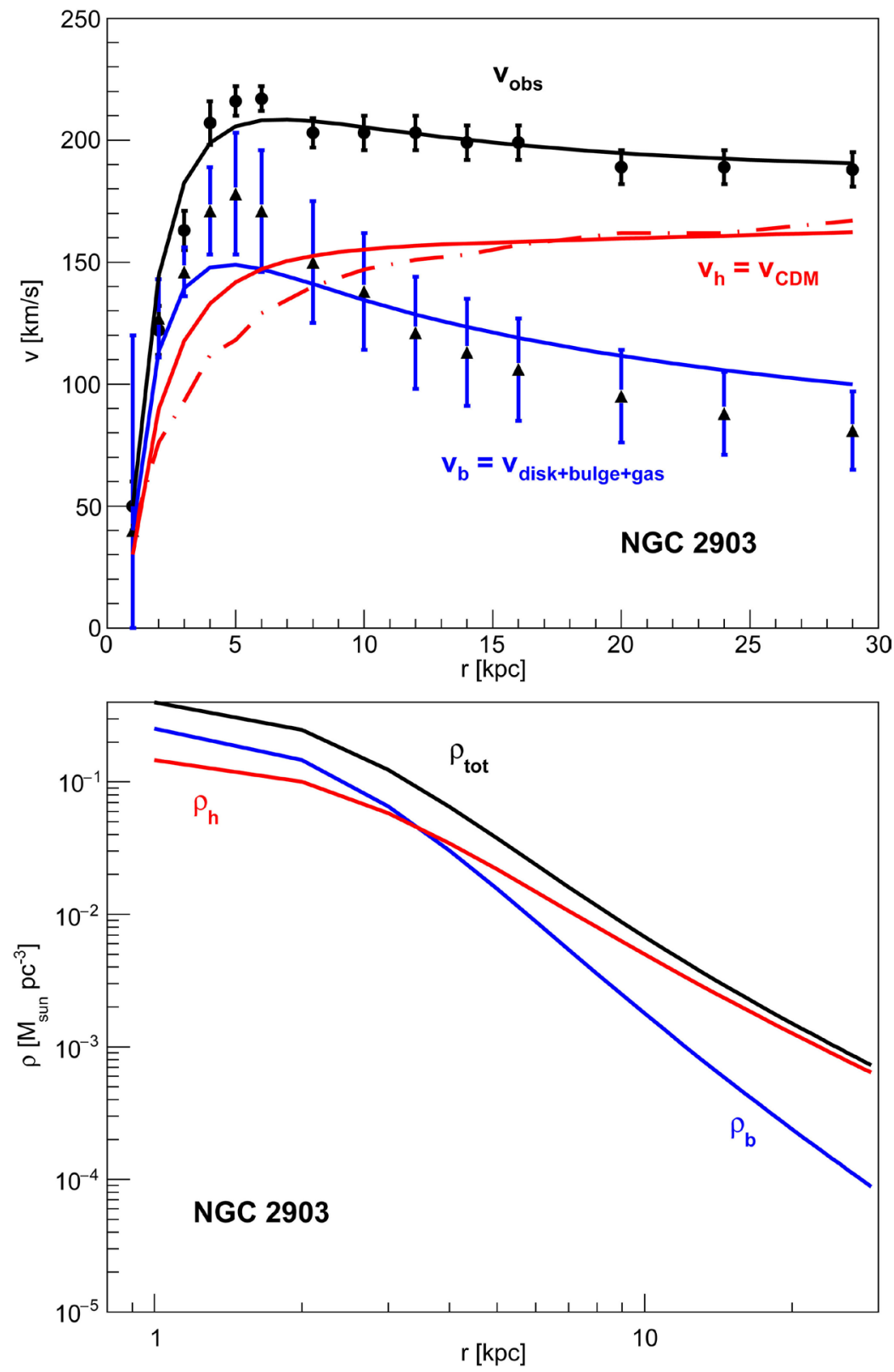

Figure 4. Top figure: Observed rotation curve $v_{\mathrm{obs}}(r)$ (dots) and the baryon contribution $v_{b}(r)$ (triangles) of galaxy NGC 2903 obtained by the THINGS collaboration [1]. The "ISO, free" fit obtains $v_{h}(r)$ (dash-dotted line) [1]. The solid lines are obtained by numerical integration as explained in the text. The fitted parameters are given in Table 1. Bottom figure: Mass densities of baryons and of dark matter of galaxy NGC 2903 obtained by numerical integration as explained in the text. 
to $1 / r^{2}$. If dark matter rotates with velocity $\sqrt{\kappa_{h}} v$ the angular momentum of dark matter of the galaxy of radius $r_{g}$ is $L_{h} \approx(\pi / 8) M_{h} \sqrt{\kappa}_{h} v r_{g}$. From the numerical integration for galaxy NGC 2403, $L_{b}=0.15 M_{b} \sqrt{\kappa_{b}} v r_{g}$ with $\kappa_{b}=0.98$. Solving for $\kappa_{h}$ we obtain $\kappa_{h} \approx 0.143$.

For galaxy NGC 3198 we obtain $L_{b}=0.16 M_{b} \sqrt{\kappa}_{b} v r_{g}$ with $\kappa_{b}=0.98$, and $\kappa_{h} \approx 0.163$.
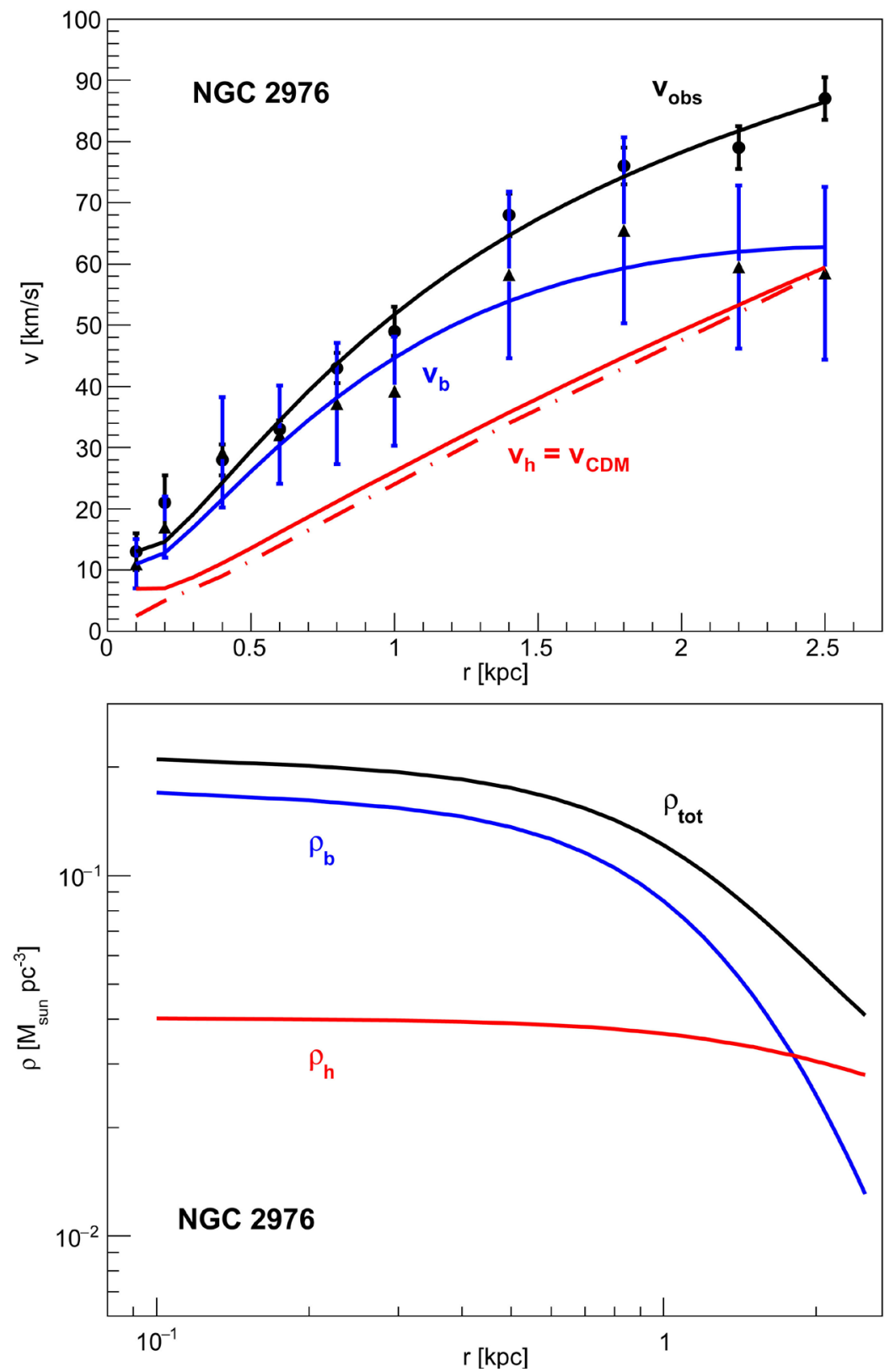

Figure 5. Top figure: Observed rotation curve $v_{\mathrm{obs}}(r)$ (dots) and the baryon contribution $v_{b}(r)$ (triangles) of galaxy NGC 2976 obtained by the THINGS collaboration [1]. The "ISO, free" fit obtains $v_{h}(r)$ (dash-dotted line) [1]. The solid lines are obtained by numerical integration as explained in the text. The fitted parameters are given in Table 1. Bottom figure: Mass densities of baryons and of dark matter of galaxy NGC 2976 obtained by numerical integration as explained in the text. 


\section{The Boltzmann Distribution}

Excellent fits to the rotation curves are obtained from Equations (3) to (6) with $\left\langle v_{r b}^{2}\right\rangle^{\prime}$ and $\left\langle v_{r h}^{2}\right\rangle^{\prime}$ independent of $r$, and non-degenerate dark matter. Since for the galaxies under consideration $\kappa_{b} \approx 1$ and $\kappa_{h} \approx 0$, we will neglect the possible dependence of $\kappa_{b}$ and $\kappa_{h}$ on $r$. From (5) and (6) we obtain
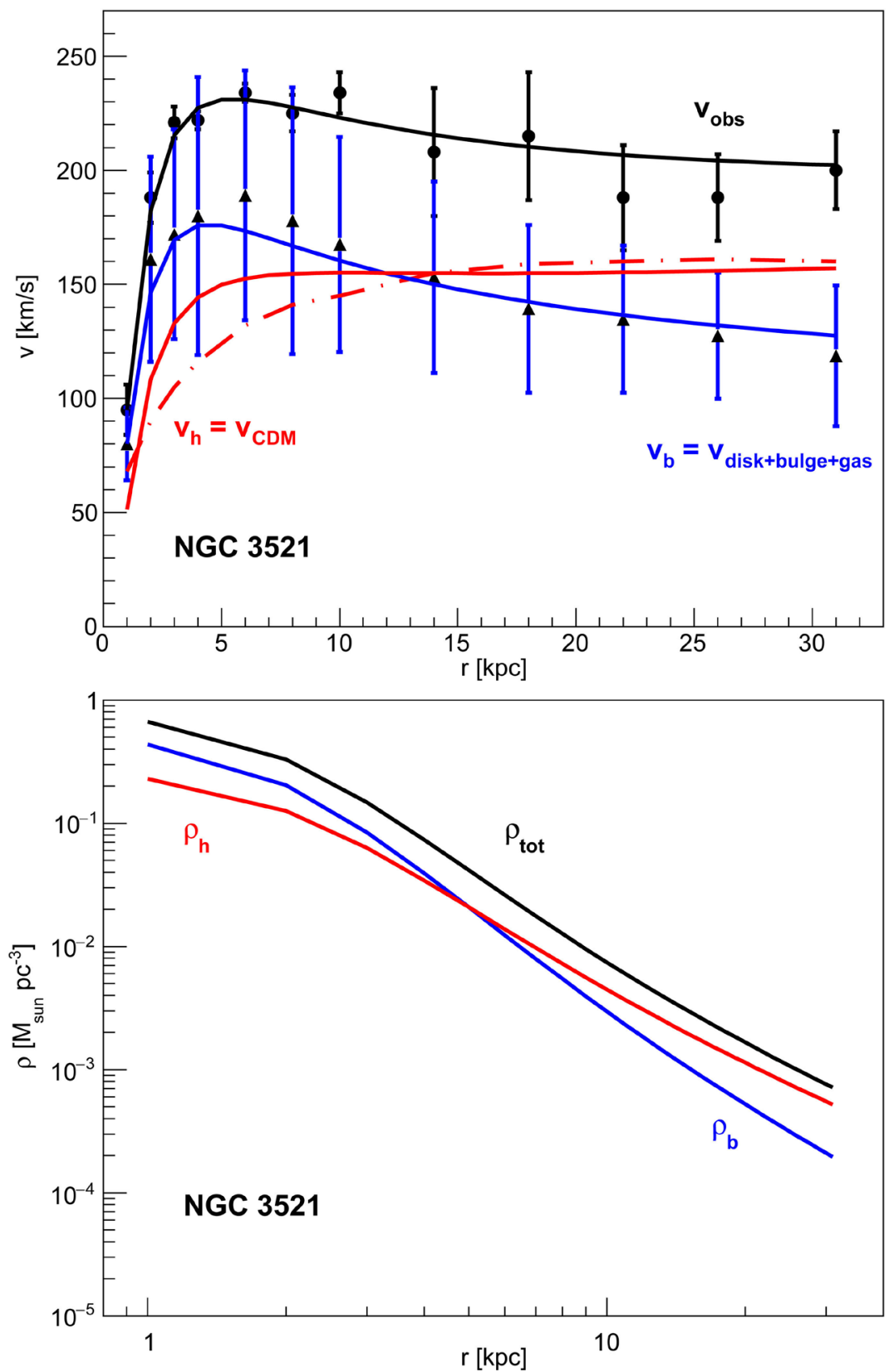

Figure 6. Top figure: Observed rotation curve $v_{\mathrm{obs}}(r)$ (dots) and the baryon contribution $v_{b}(r)$ (triangles) of galaxy NGC 3521 obtained by the THINGS collaboration [1]. The "NFW, free" fit obtains $v_{h}(r)$ (dash-dotted line) [1]. The solid lines are obtained by numerical integration as explained in the text. The fitted parameters are given in Table 1. Bottom figure: Mass densities of baryons and of dark matter of galaxy NGC $3521 \mathrm{ob}-$ tained by numerical integration as explained in the text. 


$$
\rho_{h} \propto \exp \left[\frac{-\phi(r)}{\left\langle v_{r h}^{2}\right\rangle^{\prime}}\right], \quad n_{h i}\left(r, v_{r}\right) \propto \exp \left[\frac{-v_{r}^{2} / 2-\phi(r)}{\left\langle v_{r h}^{2}\right\rangle^{\prime}}\right],
$$

where $\phi(r) \equiv-\int g(r) \mathrm{d} r$ is the gravitational potential. Note that $v_{r}^{2} / 2+\phi(r)$ is conserved. So, within the observational uncertainties of the galaxy rotation curves, dark matter satisfies the Boltzmann distribution (10). This is a non-trivial
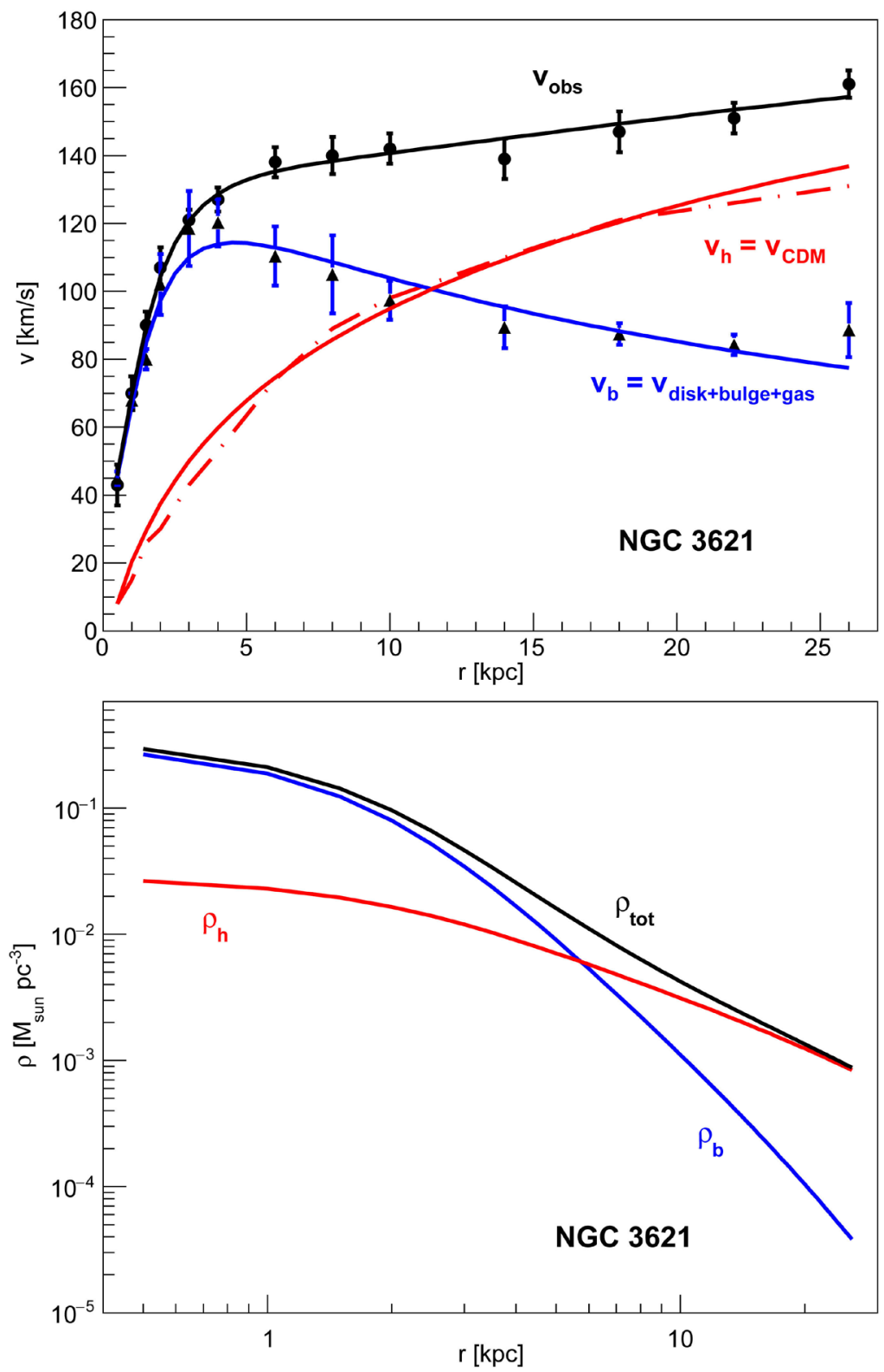

Figure 7. Top figure: Observed rotation curve $v_{\text {obs }}(r)$ (dots) and the baryon contribution $v_{b}(r)$ (triangles) of galaxy NGC 3621 obtained by the THINGS collaboration [1]. The "ISO, free" fit obtains $v_{h}(r)$ (dash-dotted line) [1]. The solid lines are obtained by numerical integration as explained in the text. The fitted parameters are given in Table 1. Bottom figure: Mass densities of baryons and of dark matter of galaxy NGC 3621 obtained by numerical integration as explained in the text. 
result. The derivation of the Boltzmann distribution in statistical mechanics assumes that the "system" under study is in "thermal equilibrium" with a "reservoir", and implies the equipartition theorem, e.g. $\frac{1}{2} m_{i} v_{i}^{2}=\frac{1}{2} m_{j} v_{j}^{2}$, which does not hold between dark matter and baryons. There is no obvious "reservoir" interacting with the dark matter, so the well known derivation of the Boltzmann
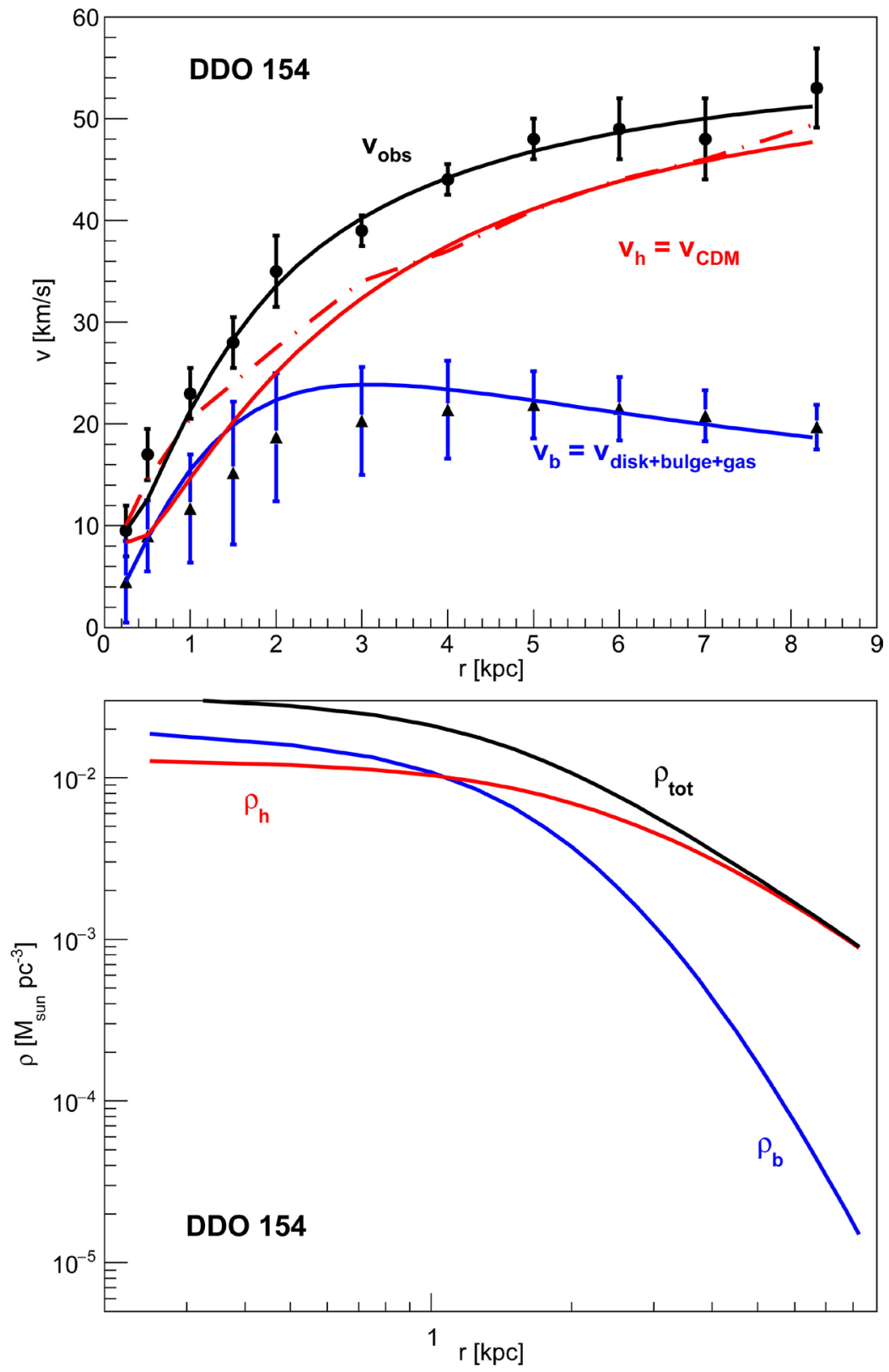

Figure 8. Top figure: Observed rotation curve $v_{\text {obs }}(r)$ (dots) and the baryon contribution $v_{b}(r)$ (triangles) of galaxy DDO 154 obtained by the THINGS collaboration [1]. The "NFW, free" fit obtains $v_{h}(r)$ (dash-dotted line) [1]. The solid lines are obtained by numerical integration for non-degenerate dark matter as explained in the text. The fitted parameters are given in Table 1. Bottom figure: Matter densities of baryons and of dark matter of galaxy DDO 154 obtained by numerical integration as explained in the text. 
distribution may not apply to dark matter, unless dark matter was once in thermal equilibrium with "something". Does the observed Boltzmann distribution suggest that dark matter was once in statistical equilibrium with the primordial (pre?) Standard Model cosmological soup?

\section{Lower Bounds on $m_{h}$ for Fermions and Bosons}

So far we have not considered the last term in Equation (6), i.e. we have set $m_{h}$
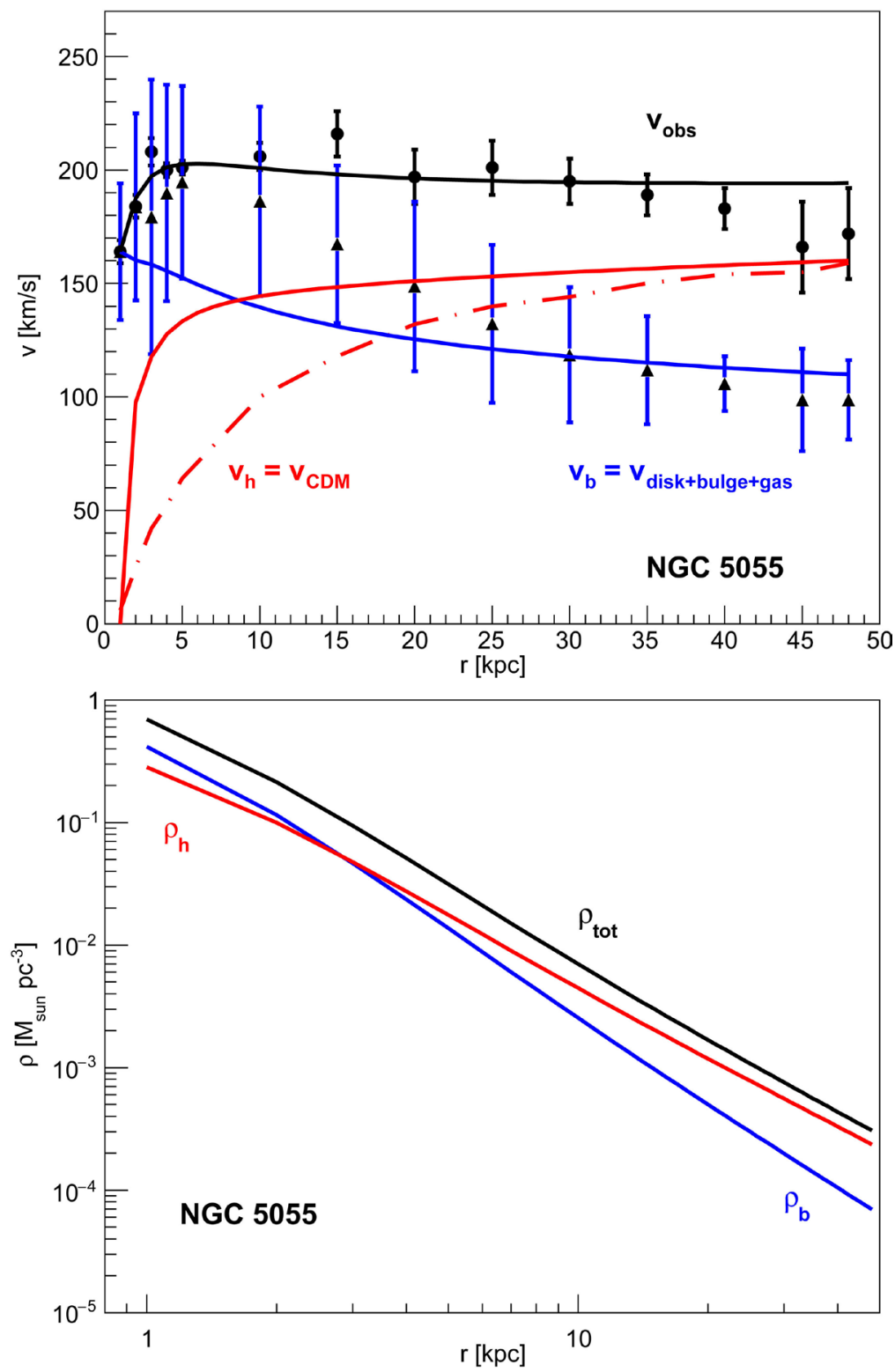

Figure 9. Top figure: Observed rotation curve $v_{\text {obs }}(r)$ (dots) and the baryon contribution $v_{b}(r)$ (triangles) of galaxy NGC 5055 obtained by the THINGS collaboration [1]. The "ISO, fixed" fit obtains $v_{h}(r)$ (dash-dotted line) [1]. The solid lines are obtained by numerical integration as explained in the text. The fitted parameters are given in Table 1. Bottom figure: Mass densities of baryons and of dark matter of galaxy NGC 5055 obtained by numerical integration as explained in the text. 
to some high value. Note that we obtain excellent fits to the galaxy rotation curves assuming $P_{h}(r)=\left\langle v_{r h}^{2}\right\rangle \rho_{h}(r)$ with $\left\langle v_{r h}^{2}\right\rangle$ independent of $r$. By lowering the value of $m_{h}$ in the fits we can probe non-linearities between $P_{h}(r)$ and $\rho_{h}(r)$. In particular, we study the onset of Fermi-Dirac or Bose-Einstein degeneracy of dark matter composed of particles of mass $m_{h}$. The dark matter density and pressure are [7]

$$
\rho_{h}=\left\langle v_{r h}^{2}\right\rangle^{3 / 2} \frac{N_{f, b} m_{h}^{4}}{(2 \pi)^{3 / 2} \hbar^{3}}\left[\frac{e^{\mu^{\prime}}}{1^{3 / 2}} \mp \frac{e^{2 \mu^{\prime}}}{2^{3 / 2}}+\frac{e^{3 \mu^{\prime}}}{3^{3 / 2}} \mp \frac{e^{4 \mu^{\prime}}}{4^{3 / 2}}+\cdots\right],
$$
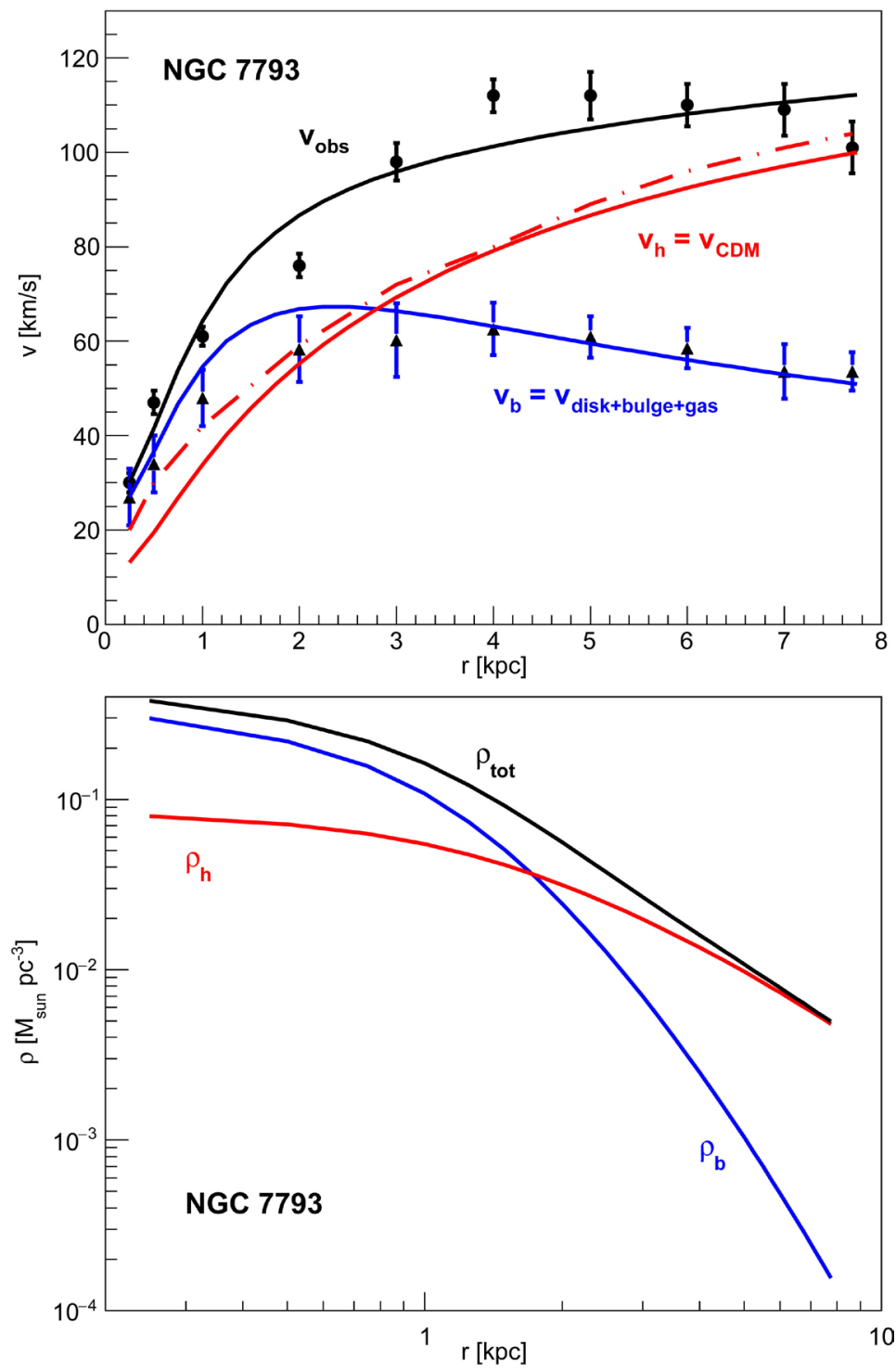

Figure 10. Top figure: Observed rotation curve $v_{\text {obs }}(r)$ (dots) and the baryon contribution $v_{b}(r)$ (triangles) of galaxy NGC 7793 obtained by the THINGS collaboration [1]. The "NFW, fixed" fit obtains $v_{h}(r)$ (dash-dotted line) [1]. The solid lines are obtained by numerical integration for non-degenerate dark matter as explained in the text. The fitted parameters are given in Table 1. Bottom figure: Mass densities of baryons and of dark matter of galaxy NGC 7793 obtained by numerical integration as explained in the text. 


$$
P_{h}=\left\langle v_{r h}^{2}\right\rangle^{5 / 2} \frac{N_{f, b} m_{h}^{4}}{(2 \pi)^{3 / 2} \hbar^{3}}\left[\frac{e^{\mu^{\prime}}}{1^{5 / 2}} \mp \frac{e^{2 \mu^{\prime}}}{2^{5 / 2}}+\frac{e^{3 \mu^{\prime}}}{3^{5 / 2}} \mp \frac{e^{4 \mu^{\prime}}}{4^{5 / 2}}+\cdots\right],
$$

where $\mu^{\prime} \equiv \mu /\left(k T_{h}\right)$, and upper signs are for fermions, and lower signs are for bosons. $\mu$ is the chemical potential. In the course of integrating Equations (3) to (6) numerically we need to obtain $\rho_{h}(r)$ given $P_{h}(r)$ and $\left\langle v_{r h}^{2}\right\rangle$. We do this by solving (12) for $e^{\mu^{\prime}}$ and substituting the result in (11). We consider up to third order terms in the series of (11) and (12) until the onset of full degeneracy for fermions:

$$
P_{h}=\rho_{h}^{5 / 3} \frac{6^{2 / 3} \pi^{4 / 3} \hbar^{2}}{5 N_{f}^{2 / 3} m_{h}^{8 / 3}},
$$

or the onset of Einstein condensation for bosons:

$$
P_{h}=\left\langle v_{r h}^{2}\right\rangle \rho_{h} \cdot 0.5136,
$$

where the numerical factor is the ratio of the two series with $\mu=0$.

For bosons $0<e^{\mu^{\prime}}<1$. Einstein condensation sets in when $e^{\mu^{\prime}}$ reaches 1 . As $m_{h}$ is lowered we find that $e^{\mu^{\prime}}$ at $r_{\min }$ may exceed 1 indicating the onset of Einstein condensation. However the $\chi^{2}$ of the fit to the galaxy rotation curves already exclude this value of $m_{h}$ even when we increase the starting value of $g\left(r_{\min }\right)=-v^{2} / r_{\min }$ within experimental bounds. In conclusion, the galaxy rotation curves exclude Einstein condensation in the studied galaxies.

Suppose a galaxy (not in Table 1) has a core with Einstein condensation. Let bosons in excited states fall to the ground state. The pressure will drop and hence more bosons will fall to smaller $r$ and condense, and the process is runaway. But this condensation and collapse does not occur in the galaxies studied in this article. Einstein condensation would show up as a divergence of $\rho_{h}$ as $r \rightarrow 0$ in Figure 1 .

Fits to rotation curves of galaxy NGC 3198 for several values of $m_{h}$ are summarized in Table 5. From this Table we conclude, with $99 \%$ confidence, that

Table 5. Fits to the rotation curves of galaxy NGC 3198 for several values of $m_{h}$ to study the onset of fermion or boson degeneracy. The four fitted parameters are $\left\langle v_{r h}^{2}\right\rangle^{\prime}$ and $\left\langle v_{r b}^{2}\right\rangle^{\prime}$ defined in Equation (9), and $\rho_{h}\left(r_{\min }\right)$ and $\rho_{b}\left(r_{\min }\right)$. The number of degrees of freedom is 22. From this table we conclude, with $99 \%$ confidence, that $m_{h}>16 \mathrm{eV}$ for fermions, and $m_{h}>45 \mathrm{eV}$ for bosons. This limit for bosons sets in before Einstein condensation at $m_{h}=35 \mathrm{eV} . \kappa_{b}=0.98, \kappa_{h}=0.15$.

\begin{tabular}{cccccccccc}
\hline \multicolumn{8}{c}{ Fermions } \\
\hline$m_{h}[\mathrm{eV}]:$ & 500 & 100 & 30 & 20 & 17 & 16 & 15 & 14 & 13 \\
$\chi^{2}:$ & 8.2 & 8.1 & 10.1 & 9.9 & 12.2 & 19.3 & 30.5 & 44.9 & 61.4 \\
\hline \multicolumn{7}{c}{ Bosons } \\
\hline$m_{h}[\mathrm{eV}]:$ & 500 & 100 & 60 & 50 & 45 & 40 & 37 & 35 & 33 \\
$\chi^{2}:$ & 8.2 & 8.3 & 10.0 & 13.6 & 17.7 & 24.9 & 31.4 & 36.3 & 41.2 \\
\hline
\end{tabular}


$m_{h}>16 \mathrm{eV}$ for fermions, and $m_{h}>45 \mathrm{eV}$ for bosons, if they dominate the dark matter density. Einstein condensation sets in at $m_{h}=35 \mathrm{eV}$.

As another example let us consider galaxy NGC 2403. For fermions, degeneracy sets in at $m_{h} \approx 40 \mathrm{eV}$, full degeneracy begins at $m_{h} \approx 30 \mathrm{eV}$, and the limit we can set based on the increase of the $\chi^{2}$ of the fit by 9 units is $m_{h}=18$ $\mathrm{eV}$. For bosons, degeneracy sets in at $m_{h} \approx 50 \mathrm{eV}$, Einstein condensation begins at $m_{h}=47 \mathrm{eV}$, and for NGC 2403 no limit on $m_{h}$ can be set based on the increase of $\chi^{2}$. Additional examples are presented in Table 4.

In conclusion, the observed spiral galaxy rotation curves disfavor dark matter dominated by axion-like bosons with mass less than $45 \mathrm{eV}$.

\section{Measurement of the Dark Matter Particle Mass $\boldsymbol{m}_{h}$}

To be specific let us consider galaxy NGC 2403. The observed rotation curves of this galaxy, presented in Figure 2, are obtained with the 4 fitted parameters listed in Table 1. In particular $\left\langle v_{r h}^{2}\right\rangle^{\prime}=101 \pm 3 \mathrm{~km} / \mathrm{s}$, and $\rho_{h}\left(r_{\min }\right)=0.075 \pm 0.014 M_{\odot} / \mathrm{pc}^{3}$. Derived parameters are presented in Table 3 . The mean dark matter density in the sphere of proper radius $r_{g}$ is $\bar{\rho}_{h}=M_{b} \Omega_{h} /\left(\Omega_{b} 4 \pi r_{g}^{3} / 3\right) \approx 0.00455 M_{\odot} / \mathrm{pc}^{3}$. We wish to relate these parameters to the primordial root-mean-square velocity of dark matter particles when perturbations were linear. We assume that dark matter particles have decoupled while still ultrarelativistic, i.e. have negligible interactions besides gravity. We take $\kappa_{h} \approx 0.15$.

A model of the hierarchical formation of galaxies is described in References [5] and [6]. Consider the expanding sphere of proper radius $r_{g}$ and comoving radius $r_{c}$ at time $t_{g}$ containing the matter that will form galaxy NGC 2403. This sphere expands, reaches maximum expansion, and then collapses to form galaxy NGC 2403, see figure 5 of Reference [6]. We estimate the root-mean-square velocity of dark matter at time $t_{g}$, when perturbations are still linear, to be

$$
v_{h \mathrm{rms}} \approx \sqrt{3}\left\langle v_{r h}^{2}\right\rangle^{1 / 2}\left(\frac{\bar{\rho}_{h}}{\rho_{h}\left(r_{\min }\right)}\right)^{1 / 3} \approx 63 \mathrm{~km} / \mathrm{s} .
$$

The absolute velocity $v_{h r m s}$ has contributions from three spatial components, hence the factor $\sqrt{3}$. The last factor is a correction corresponding to adiabatic compression. According to the model of the hierarchical formation of galaxies, Equation (15) should be valid even with merging of galaxies. A correction due to mixing, expected to be of order $\mathrm{O}(1)$, may be needed. Extrapolating to the past, we find that the expansion parameter at which dark matter particles become non-relativistic is

$$
a_{h \mathrm{NR}} \approx \frac{r_{g}}{r_{c}} \frac{v_{h \mathrm{rms}}}{c} \approx 4.1 \times 10^{-6},
$$

and the corresponding photon temperature is $T_{0} / a_{h \mathrm{NR}} \approx 57 \mathrm{eV}$. Note that $v_{h r m s} \propto 1 / a$ for non-relativistic particles, and the expansion parameter at time $t_{g}$ is $a=r_{g} / r_{c}$. Equation (16) assumes that dark matter particles decoupled while still ultra-relativistic. To be specific, we assume that particles of mass $m_{h}$ 
dominate dark matter, and that these particles have $N_{b}=0$ boson degrees of freedom, and $N_{f}=2$ fermion degrees of freedom. The number density of dark matter particles at expansion parameter $a_{h \mathrm{NR}}$ can be calculated in two ways:

$$
n_{h \mathrm{NR}}=\frac{\Omega_{c} \rho_{\text {crit }}}{m_{h} a_{h \mathrm{NR}}^{3}}=\frac{1.20205}{\pi^{2}}\left(\frac{k T_{h \mathrm{NR}}}{\hbar c}\right)^{3}\left\{N_{b}+\frac{3}{4} N_{f}\right\},
$$

where the ultra-relativistic expression to the right assumes zero chemical potential. Substituting $m_{h} c^{2} \approx 3.15 k T_{h \mathrm{NR}}$, we obtain $m_{h} \approx 70 \mathrm{eV}$. The ratio of the temperatures of dark matter and photons is $T_{h} / T \approx m_{h} /(3.15 \times 57 \mathrm{eV}) \approx 0.39$ after $e^{+} e^{-}$annihilation while dark matter remains ultra-relativistic. Repeating these calculations for other galaxies, and including statistical uncertainties derived from the uncertainties of the measured galaxy rotation curves, we obtain Table 6.

Note that $M_{b}$ and $r_{g}$ drop out of the expression for $a_{h \mathrm{NR}}$ :

$$
a_{h \mathrm{NR}}=\frac{\sqrt{3}\left\langle v_{r h}^{2}\right\rangle^{1 / 2}}{c}\left[\frac{\Omega_{c} \rho_{\text {crit }}}{\rho_{h}\left(r_{\text {min }}\right)}\right]^{1 / 3},
$$

with $r_{\min } \rightarrow 0$. This equation can be understood directly: it expresses $\left\langle v_{r h}^{2}\right\rangle^{1 / 2} \propto 1 / a$, were the last factor in (18) is the "expansion parameter" in the core of the galaxy.

Table 6. Measurements of the expansion parameter $a_{h \mathrm{NR}}$ at which dark matter becomes non-relativistic (calculated with Equation (18), and the fits in Table 1). All uncertainties are statistical, $1 \sigma$. The parameter correlation coefficient -0.802 between $\left\langle v_{r h}^{2}\right\rangle^{1 / 2}$ and $\rho_{h}\left(r_{\min }\right)$ is taken into account. Also shown are two parameters derived from $a_{h \mathrm{NR}}$ : the dark matter particle mass $m_{h}$, and the ratio of temperatures $T_{h} / T$ of dark matter and photons after $e^{+} e^{-}$annihilation and before dark matter becomes non-relativistic. ${ }^{*} \mathrm{Ga}$ laxy NGC 2841 was excluded from the average because the measurement of $\rho_{h}(\mathrm{~min})$ does not reach the core, see Figure 3. The uncertainty of the average is multiplied by $\left[\chi^{2} /(9-1)\right]^{1 / 2}=2.17$ (as recommended in [2]).

\begin{tabular}{cccc}
\hline Galaxy & $10^{6} \times a_{h \mathrm{NR}}$ & $m_{h}[\mathrm{eV}]$ & $T_{h} / T$ \\
\hline NGC 2403 & $4.09 \pm 0.38$ & $70.0 \pm 4.9$ & $0.387 \pm 0.009$ \\
NGC 2841* & $8.29 \pm 0.33$ & $41.2 \pm 1.3$ & $0.462 \pm 0.006$ \\
NGC 2903 & $4.60 \pm 0.32$ & $64.1 \pm 3.4$ & $0.399 \pm 0.008$ \\
NGC 2976 & $6.44 \pm 2.33$ & $49.8 \pm 13.5$ & $0.433 \pm 0.039$ \\
NGC 3198 & $4.99 \pm 0.44$ & $60.3 \pm 4.0$ & $0.407 \pm 0.010$ \\
NGC 3521 & $4.27 \pm 0.82$ & $67.8 \pm 9.7$ & $0.391 \pm 0.019$ \\
NGC 3621 & $7.26 \pm 0.76$ & $45.5 \pm 3.6$ & $0.447 \pm 0.012$ \\
DDO 154 & $2.65 \pm 0.47$ & $96.9 \pm 13.0$ & $0.347 \pm 0.016$ \\
NGC 5055 & $3.75 \pm 0.41$ & $74.7 \pm 6.1$ & $0.379 \pm 0.011$ \\
NGC 7793 & $3.39 \pm 0.43$ & $80.6 \pm 7.6$ & $0.369 \pm 0.012$ \\
Average & $4.17 \pm 0.34$ & $69.0 \pm 4.2$ & $0.389 \pm 0.008$ \\
\hline
\end{tabular}


Alternatively, (18) expresses adiabatic expansion of a non-relativistic ideal "noble" gas: $T_{h} V^{\gamma-1}=$ constant with $\gamma=5 / 3$. By "noble" we mean that collisions (if any) between dark matter particles do not excite internal degrees of freedom (if any) of these particles.

The independent measurements of $m_{h}$ presented in Table 6 are fairly consistent, considering that the masses $M_{b}$ of the galaxies span three orders of magnitude, and the angular momenta $L_{b}$ span five orders of magnitude. The average over nine independent measurements presented in Table 6 is

$$
a_{h \mathrm{NR}}=[4.17 \pm 0.34(\text { stat })] \times 10^{-6}, \quad m_{h}=69.0 \pm 4.2(\text { stat }) \mathrm{eV},
$$

and the average $T_{h} / T$, after $e^{+} e^{-}$annihilation while dark matter is still ultra-relativistic, is

$$
T_{h} / T=0.389 \pm 0.008 \text { (stat), }
$$

where $T$ is the photon temperature. Note that $m_{h} \propto a_{h \mathrm{NR}}^{-3 / 4}$ and $T_{h} / T \propto a_{h \mathrm{NR}}^{1 / 4}$, so the correlated uncertainties of $m_{h}$ and $T_{h} / T$ can be derived directly from the uncertainty of $a_{h \mathrm{NR}}$.

The near coincidence of the two temperatures $T_{h}$ and $T$ is evidence that dark matter was once in thermal equilibrium with the (pre?) Standard Model particles, which explains the observed Boltzmann distribution. From the preceding analysis we also conclude that dark matter decoupled while still ultra-relativistic, so scenarios with freeze-out, or freeze-in are disfavored.

Let us summarize. We assume that ultra-relativistic dark matter has zero chemical potential, and obtain, from the spiral galaxy rotation curves, a value of $T_{h} / T$ that is consistent with thermal equilibrium of dark matter with the Standard Model sector at some time in the early Universe. This is a highly significant result since $T$ depends on $T_{0}$ while $T_{h}$ does not. If we would have obtained a different value of the "adiabatic invariant" $\left\langle v_{r h}^{2}\right\rangle^{1 / 2} \rho_{h}\left(r_{\min }\right)^{-1 / 3}$, we would have concluded that the chemical potential of dark matter is different from zero, and/or dark matter was never in thermal equilibrium with the Standard Model sector. So the observed adiabatic invariant is strong evidence that dark matter has zero chemical potential and was once in thermal equilibrium with the Standard Model particles.

\section{Estimate of Systematic Uncertainties}

The statistical uncertainties (at $68 \%$ confidence) presented so far are derived from the observational uncertainties of the galaxy rotation curves, and contributions from $h$ and $\Omega_{c}$.

The main systematic uncertainty comes from Equation (15). This Equation is justified by the model of the hierarchical formation of galaxies [5] [6], and should be valid even for merging galaxies. Never-the-less Equation (15) may require a correction due to mixing. Consider galaxy NGC 3198. The time for a particle of dark matter to traverse the radius $r_{g}$ is $1.3 \mathrm{Gyr}$, so mixing due to particles entering and leaving the sphere occupied by the galaxy may be impor- 
tant. At time $t_{g}$ mixing can be neglected because the Universe is nearly homogeneous. Mixing during expansion and contraction can also be neglected. Most mixing occurs after the galaxy has formed. Even then, particles leaving and entering the sphere of proper radius $r_{g}$ are expected to have approximately the same $\left\langle v_{r h}^{2}\right\rangle$, exept for a correction due to the expansion of the Universe. So we expect the correction due to mixing to be $\mathrm{O}(1)$ for galaxies in a stationary state, as confirmed by the fair consistency of measurements with galaxies with masses that span three orders of magnitude, and angular momenta that span five orders of magnitude. Further study of this issue is needed.

Another correction of $\mathrm{O}(1)$ is due to the non-spherical symmetry of the galaxies. Yet another correction of $\mathrm{O}(1)$ may come from the details of the transition from ultra-relativistic to non-relativistic dark matter.

Pending detailed studies, our preliminary estimates of systematic uncertainties are:

$$
\begin{gathered}
a_{h \mathrm{NR}}=[4.17 \pm 0.34(\text { stat }) \pm 2.50(\text { syst })] \times 10^{-6}, \\
m_{h}=69.0 \pm 4.2(\text { stat }) \pm 31.0(\text { syst }) \mathrm{eV}, \\
T_{h} / T=0.389 \pm 0.008(\text { stat }) \pm 0.058(\text { syst }) .
\end{gathered}
$$

Note that (22) and (23) follow directly from (21): their uncertainties are correlated. In comparison, the current uncertainty of $m_{h}$ spans 70 orders of magnitude for fermions, and 90 orders of magnitude for bosons [8].

\section{Thermalized Dark Matter That Decouples While Ultra-Relativistic}

We consider dark matter that at some time in the history of the Universe was in thermal equilibrium with the (pre?) Standard Model particles, and decoupled from these particles and from self-annihilation while still ultra-relativistic. To be specific, we consider dark matter to be dominated by a single family of sterile Majorana neutrinos, i.e. $N_{b}=0$ boson degrees of freedom, and $N_{f}=2$ fermion degrees of freedom (for spin-up and spin-down). We also assume three families of active Majorana neutrinos with $N_{f}=2$ each.

As the universe expands and cools, Standard Model particles and antiparticles that become non-relativistic annihilate heating the Standard Model sector conserving entropy, without heating sterile and active neutrinos if they have already decoupled. Table 7 presents the ratio $T_{h} / T$ of dark matter-to-photon temperatures after $e^{+} e^{-}$annihilation while dark matter is still ultra-relativistic. Also presented is the value of $m_{h}$ needed to obtain the measured $\Omega_{c}$. We note that the measured $T_{h} / T$ (23) and measured $m_{h}$ (22) are consistent with decoupling at temperatures higher than approximately $m_{\mu}$.

For example, assume the decoupling temperature is in the range $m_{b}$ to $m_{W}$. Then $T_{h} / T$ is calculated as follows:

$$
\frac{T_{h}}{T}=\left(\frac{4 \times 43}{11 \times 345}\right)^{1 / 3}=0.357 .
$$


Table 7. We consider dark matter that was once in thermal equilibrium with the (pre?) Standard Model particles and then decoupled from these particles in the indicated temperature range. We assume no self-annihilation. $T_{h} / T$ is the ratio of dark matter temperature to photon temperature after $e^{+} e^{-}$annihilation while dark matter remains ultra-relativistic. $m_{h}$ is the mass of particles of dark matter needed to obtain the observed $\Omega_{c}$. Case $N_{b}=0, N_{f}=2$.

\begin{tabular}{ccc}
\hline Decoupling temperature range & $T_{h} / T$ in range $m_{h}$ to $m_{e}$ & $m_{h}$ \\
\hline$m_{H}$ to $m_{t}$ & 0.344 & $99.9 \pm 3.1 \mathrm{eV}$ \\
$m_{W}$ to $m_{H}$ & 0.345 & $98.9 \pm 3.1 \mathrm{eV}$ \\
$m_{b}$ to $m_{W}$ & 0.357 & $89.5 \pm 2.8 \mathrm{eV}$ \\
$m_{\tau}$ to $m_{b}$ & 0.372 & $78.6 \pm 2.5 \mathrm{eV}$ \\
$m_{c}$ to $m_{\tau}$ & 0.378 & $75.0 \pm 2.4 \mathrm{eV}$ \\
$m_{s}$ to $m_{c}$ & 0.399 & $64.1 \pm 2.0 \mathrm{eV}$ \\
$m_{e}$ to $m_{\mu}$ & 0.714 & $11.2 \pm 0.4 \mathrm{eV}$ \\
\hline
\end{tabular}

These numbers can be found in the Table in Section 21.3.2 of [2]. At the time of Big Bang Nucleosynthesis (BBN), i.e. $T \approx 1 \mathrm{MeV}$, neutrinos with, for example, $m_{h}=89.5 \pm 2.8 \mathrm{eV}$ are still ultra-relativistic. The density of radiation at the time of BBN increases by a factor

$$
\frac{2+\frac{7}{8} 6\left(\frac{4}{11}\right)^{4 / 3}+\frac{7}{8} 2\left(\frac{4 \times 43}{11 \times 345}\right)^{4 / 3}}{2+\frac{7}{8} 6\left(\frac{4}{11}\right)^{4 / 3}}=\frac{3.391}{3.363}=1.0084,
$$

due to one family of sterile Majorana neutrinos. Equivalently, the effective number of light neutrinos increases from $N_{v}=3$ to 3.062, well within BBN experimental bounds, i.e. $N_{v} \lesssim 4 \quad$ [2].

An example of a sterile neutrino dark matter candidate is presented in Figure 11. The figure was obtained by integrating the corresponding Boltzmann equation for the generation of sterile Majorana neutrinos since Electroweak Symmetry Breaking (when active Majorana neutrinos acquire a Dirac mass and therefore sterile Majorana neutrinos couple to the Standard Model). Figure 11 also shows a brief history of the Universe.

\section{Dark Matter-Baryon Cross-Section}

Consider a hydrogen atom, or a proton, of mass $m_{p}$, in a circular orbit of velocity $V_{b}$ within the core of a galaxy. The velocity $V_{b}$ will decay with a relative rate $\tau_{b}^{-1} \equiv \mathrm{d} V_{b} /\left(V_{b} \cdot \mathrm{d} t\right) \approx \rho_{h} \sigma_{h b} V_{b} / m_{p}$ due to collisions with dark matter particles with cross-section $\sigma_{h b}$. Taking $\tau_{b}$ equal to the age of the universe, $V_{b} \approx 150 \mathrm{~km} / \mathrm{s}$, and $\rho_{h} \approx 0.2 M_{\odot} / \mathrm{pc}^{3} \quad$ (see Table 1), we obtain

$$
\sigma_{h b} \lesssim 2 \times 10^{-26} \mathrm{~cm}^{2}
$$




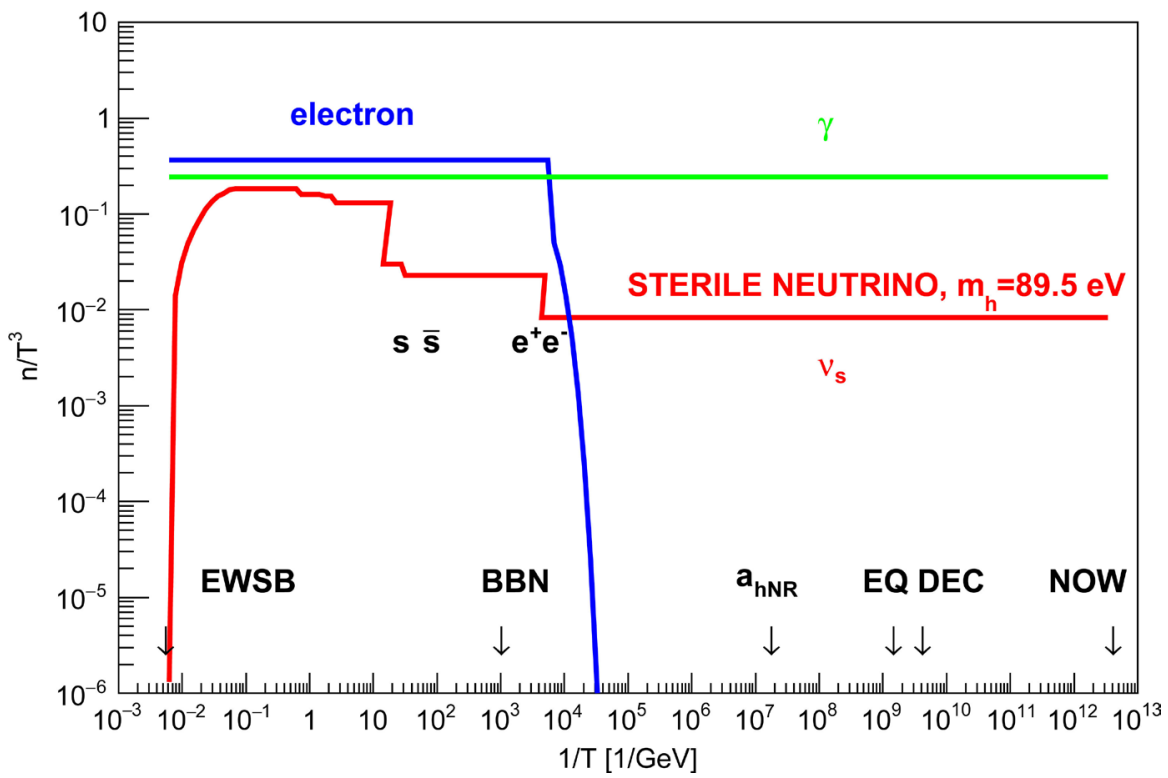

Figure 11. Plotted are the number density $n$ of photons, electrons, and dark matter particles, divided by $T^{3}$, as a function of $1 / T$, where $T$ is the photon temperature. In this example, the dark matter is dominated by one Majorana sterile neutrino type (two degrees of freedom: spin up, and spin down) with $m_{s}=89.5 \mathrm{eV}$, produced since Electroweak Symmetry Breaking (EWSB) at $T=159 \pm 1 \mathrm{GeV}$. The corresponding active neutrino flavor in this example has $m_{a}=1 \times 10^{-9} \mathrm{eV}$, but this value is not critical. The sterile neutrino in this example enters thermal equilibrium at $T \approx 53 \mathrm{GeV}$, and leaves thermal contact at $T \approx 6 \mathrm{GeV}$. The mass $m_{s}=89.5 \mathrm{eV}$ is chosen to obtain the observed $\Omega_{c}$. This solution is consistent with Big Bang Nucleosynthesis. The lifetime of this sterile neutrino is $7 \times 10^{27}$ years.

independently of $m_{h}$.

The mean time between collisions of a dark matter particle with baryons is $\tau_{h} \approx m_{p} /\left(\rho_{b} \sigma_{h b} \sqrt{3}\left\langle v_{r h}^{2}\right\rangle^{1 / 2}\right)$. Dark matter is not in thermal equilibrium with baryons so $\tau_{h}$ is larger than the age of the universe. With data of galaxy NGC 2841 we obtain

$$
\sigma_{h b} \lesssim 2 \times 10^{-26} \mathrm{~cm}^{2} .
$$

These limits are useful for dark matter masses $m_{h}$ below the reach of direct or indirect searches, e.g. $m_{h}<50 \mathrm{keV}$. Planed direct low mass WIMP searches with the NEWS-SNO project will reach dark matter masses $\approx 0.1 \mathrm{GeV}$ [2]. No searches in the $m_{h}=100 \mathrm{eV}$ range are mentioned in the Dark Matter review of Reference [2], but ideas to reach the $\mathrm{MeV}$ to $\mathrm{meV}$ mass scales are mentioned.

\section{The Core}

The mean baryon density of the universe is $6.2 \times 10^{-9} M_{\odot} / \mathrm{pc}^{3}$, while the baryon density in the core of galaxy NGC 2403 is $0.3 M_{\odot} / \mathrm{pc}^{3}$, so matter has collapsed by a factor $5 \times 10^{7}$ in this case. What prevented $\rho_{h}(r)$ or $\rho_{b}(r)$ from collapsing all the way to $\infty$ as $r \rightarrow 0$ ? In Figures 1-10 we see no increase of $\rho_{h}(r)$ or $\rho_{b}(r)$ as $r \rightarrow 0$ down to the first measured point at $r_{\min }$. Why a 
core instead of a cusp? The four parameters $\rho_{h}\left(r_{\min }\right), \rho_{b}\left(r_{\min }\right),\left\langle v_{r h}^{2}\right\rangle^{\prime}$, and $\left\langle v_{r b}^{2}\right\rangle^{\prime}$ can fit any core radius. So, what determines these four boundary conditions?

Possibilities to consider are Bose-Einstein of Fermi-Dirac degeneracy? (the observed galaxy rotation curves obtain $m_{h}$ at the edge of degeneracy as shown in Section 7), dark matter or baryon crossing time?, non-spherical initial density perturbations?, angular momentum of the primordial rotation field?, dominance of baryons in the core?, ongoing collapse of the core? (however baryons are already supported by the centrifugal force as shown in Section 4), etc.

In Figures 1-10 we observe that the baryon core radius, the radius $r_{\text {ed }}$ of equal densities, and the dark matter core radius are similar, and increase in this order. We also observe that the baryon density dominates at small radii. These observations suggest that baryons radiate energy conserving angular momentum, fall to the galactic plane, acquire increasingly circular orbits, until baryons are supported by the centrifugal force with $\kappa_{b} \rightarrow 1$. Thereafter baryons can fall no further. For example, a proton or hydrogen atom falling into the galaxy collides with other baryons, falls to the disk and acquires circular velocity $\sqrt{\kappa_{b}} v$. Once the central gravitational potential is determined mostly by baryons, dark matter rests in this given potential.

Note that as $r \rightarrow \infty, \rho_{h} \rightarrow v^{2} /\left(4 \pi G r^{2}\right)$ with $v^{2}=2\left\langle v_{r h}^{2}\right\rangle^{\prime}$. Note that (18) obtains $\rho_{h}(0)$ given $\left\langle v_{r h}^{2}\right\rangle$ and $a_{h \mathrm{NR}}$, and therefore constrains the dark matter core radius.

\section{Angular Momentum}

The suggestion is that the primordial angular momentum in the sphere of proper radius $r_{g}$ and comoving radius $r_{c}$ during the expansion phase at time $t_{g}$ is the same as the angular momentum $L=L_{b}\left(\Omega_{c}+\Omega_{b}\right) / \Omega_{b}$ of the galaxy with $L_{b}$ listed in Table 4, i.e. the angular momentum of the galaxy is conserved and may be of cosmological origin.

Perturbations of density, gravitational potential, and velocity in the non-relativistic universe dominated by dark matter have four independent modes [9]: a scalar growing mode, a scalar decaying mode, and two vector modes with transverse velocity $\left(\boldsymbol{q} \cdot \delta \boldsymbol{v}_{\boldsymbol{q}}=0\right.$ in Fourier space) that conserve angular momentum, i.e. $\delta v_{q} \propto 1 / a$. The velocity field of these vector modes is measured by the galaxy angular momenta listed in Table 4 if they are of cosmological origin.

\section{Structure Formation}

Most dark matter should have been non-relativistic at the time when there was a galactic mass inside the horizon [2]. From this criterion we have estimated $m_{h} \gtrsim 1.3 \mathrm{keV}$ for unsuppressed formation of galaxies with total (baryonic plus dark) mass as low as $10^{8} M_{\odot}[10]$. On the other hand, simulations of the Cold Dark Matter scenario produce many more dwarf galaxies than observed [11].

At an expansion parameter $a_{h \mathrm{NR}} \approx 4.2 \times 10^{-6}$, when dark matter with mass 
$m_{h} \approx 69 \mathrm{eV}$ becomes non-relativistic, the universe is still dominated by radiation, see Figure 11. The age of the universe is $t=a_{h \mathrm{NR}}^{2} /\left(2 H_{0} \sqrt{\Omega_{r}}\right)$, the distance to the horizon is $l=2 c t$, and the mass of baryons inside the horizon is $M_{b} \approx 20 \times 10^{10} M_{\odot}$, comparable to large galaxies in Table 3. Smaller size perturbations are suppressed, so we may have a problem understanding the formation of galaxies with $M_{b}<20 \times 10^{10} M_{\odot}$.

Note that protons are non-relativistic when the smallest observed galaxies and globular clusters enter the horizon. Compared to the cold dark matter scenario with $m_{h} \gg 5 \mathrm{keV}$, for $m_{h}=69 \mathrm{eV}$ the power spectrum of density fluctuations on scales smaller than $M_{b}=20 \times 10^{10} M_{\odot}$ becomes suppressed. If the dark matter inside the horizon becomes nearly homogeneous while ultra-relativistic (as expected), then the suppression factor is $\Omega_{b} /\left(\Omega_{b}+\Omega_{c}\right) \approx 0.16$ assuming adiabatic initial conditions.

The hierarchical formation of galaxies is shown in figure 6 of Reference [6]. Suppose that only the small scale perturbations are suppressed by a factor 0.16 . Since some galaxies with $M_{b} \approx 20 \times 10^{10} M_{\odot}$ have formed, other perturbations on this scale are on the verge of formation, and hence even low amplitude small scale perturbations can collapse and form a small galaxy or globular cluster. This observation may address the "missing satellite problem" [11]. A dedicated study of this issue is needed.

Dark matter with mass $\approx 69 \mathrm{eV}$ also has tension with studies of the power spectra of density perturbations on scales less than $10 \mathrm{~h}^{-1} \mathrm{Mpc}$ with the "Lyman-alpha forest" [12]. These studies set lower bounds on dark matter mass of $\approx$ $4 \mathrm{keV}$ for early decoupled thermal relics. So we have tension between these two measurements which needs further study. Note however that $69 \mathrm{eV}$ is near the limit of the reach of the Lyman-alpha forest study [12].

\section{Conclusions}

We conclude that the rotation curves of regular spiral galaxies are described, within observational uncertainties, by Equations (3) to (6). These equations describe the stationary state of two self-gravitating non-relativistic ideal gases, "baryons" and "dark matter", that do not interact significantly with each other except for gravity. These equations can be integrated numerically given four parameters (that are boundary conditions). These four parameters are obtained by minimizing a $\chi^{2}$ between the measured and calculated spiral galaxy rotation curves.

From these studies, we obtain a wide range of quantitative results on the physics of galaxies, dark matter, and cosmology. In particular, we have presented precision measurements of the dark matter mass $m_{h}$ and temperature $T_{h}(a)$.

The most significant result is the ratio of the temperatures of dark matter and photons after $e^{+} e^{-}$annihilation while dark matter is still ultra-relativistic:

$$
T_{h} / T=0.389 \pm 0.008 \text { (stat) } \pm 0.058 \text { (syst). }
$$

The plausibility that this result is correct lies in the consistency of nine com- 
plete and independent measurements of the mass $m_{h}$ of particles of dark matter with spiral galaxies that span three orders of magnitude in mass, and five orders of magnitude in angular momentum. The ratio (28) could have been orders of magnitude larger than, or smaller than, unity, because $T$ depends on $T_{0}$, while $T_{h}$ is independent of $T_{0}$. The ratio $T_{h} / T$ depends on the measured adiabatic invariant $\left\langle v_{r h}^{2}\right\rangle^{1 / 2} \rho_{h}\left(r_{\min }\right)^{-1 / 3}$, so this analysis provides evidence that dark matter was once in thermal equilibrium with the (pre?) Standard Model particles. It is therefore likely that dark matter particles will have one of the precise masses listed in Table 7 (or in its extensions for other $N_{b}$ or $N_{f}$ ). The decoupling of dark matter from the Standard Model sector at temperatures higher than $m_{\mu}$ allows us to follow the Boltzmann distribution of these particles all the way from primordial times into the cores of spiral galaxies. An integration of the Boltzmann equation for the production of sterile Majorana neutrinos after Electroweak Symmetry Breaking shows consistency with these results, see Figure 11. Such sterile Majorana neutrinos evade all current dark matter searches.

The present studies pose new questions. Why is the spiral galaxy core radius similar in galaxies that span three orders of magnitude in mass? It seems likely that this core radius is of cosmological origin. In fact Equation (18) is already a constraint. It also seems likely that the angular momentum of spiral galaxies is of cosmological origin. Studies of angular momentum correlation between galaxies should settle this issue. If the angular momenta are cosmological, we have a handle on the amplitude of the primordial vector modes. The importance of this avenue of research lies in the fact that the velocities of these vector modes grow towards the past conserving angular momentum and hence could dominate the origin of the Universe.

\section{Conflicts of Interest}

The author declares no conflicts of interest regarding the publication of this paper.

\section{References}

[1] de Blok, W.J.G., et al. (2008) High-Resolution Rotation Curves and Galaxy Mass Models from THINGS. The Astronomical Journal, 136, 2648-2719.

[2] Tanabashi, M., et al. (Particle Data Group) (2018) The Review of Particle Physics. Physical Review D, 98, Article ID: 030001.

[3] Begeman, K. (1987) HI Rotation Curves of Spiral Galaxies. PhD Thesis, University of Groningen, Groningen.

[4] Navarro, J.F., Frenk, C.S. and White, S.D.M. (1996) The Structure of Cold Dark Matter Halos. ApJ, 462, 563.

Navarro, J.F., Frenk, C.S. and White, S.D.M. (1997) A Universal Density Profile from Hierarchical Clustering. ApJ, 490, 493.

[5] Hoeneisen, B. (2000) A Simple Model of the Hierarchical Formation of Galaxies. arXiv:astro-ph/0009071. 
[6] Hoeneisen, B. (2018) Study of Galaxy Distributions with SDSS DR14 Data and Measurement of Neutrino Masses. International Journal of Astronomy and Astrophysics, 8, 230-257. https://doi.org/10.4236/ijaa.2018.83017

[7] Hoeneisen, B. (1993) Thermal Physics. Mellen Research University Press, San Francisco.

[8] Profumo, S. (2017) Particle Dark Matter. World Scientific, Singapore.

[9] Weinberg, S. (2008) Cosmology. Oxford University Press, Oxford.

[10] Hoeneisen, B. (2015) Trying to Understand Dark Matter. arXiv:1502.07375

[11] Klypin, A.A., Kravtsov, A.V., Valenzuela, O. and Prada, F. (1999) Where Are the Missing Galactic Satellites? Astrophysical Journal, 522, 8292. https://doi.org/10.1086/307643

[12] Baur, J., Palanque-Delabrouille, N., Yche, C., Magneville, C. and Viel, M. (2016) Lyman-Alpha Forests Cool Warm Dark Matter. Journal of Cosmology and Astroparticle Physics, No. 8, Article ID: 012.

https://doi.org/10.1088/1475-7516/2016/08/012 